\title{
Matrices connected with Brauer's centralizer algebras *
}

\author{
Mark D. McKerihan ${ }^{\dagger}$ \\ Department of Mathematics \\ University of Michigan \\ Ann Arbor, MI 48109
}

Submitted: October 9, 1995; Accepted: October 31,1995

\begin{abstract}
In a 1989 paper [HW1], Hanlon and Wales showed that the algebra structure of the Brauer Centralizer Algebra $A_{f}^{(x)}$ is completely determined by the ranks of certain combinatorially defined square matrices $Z^{\lambda / \mu}$, whose entries are polynomials in the parameter $x$. We consider a set of matrices $M^{\lambda / \mu}$ found by Jockusch that have a similar combinatorial description. These new matrices can be obtained from the original matrices by extracting the terms that are of "highest degree" in a certain sense. Furthermore, the $M^{\lambda / \mu}$ have analogues $\mathcal{M}^{\lambda / \mu}$ that play the same role that the $Z^{\lambda / \mu}$ play in $A_{f}^{(x)}$, for another algebra that arises naturally in this context.

We find very simple formulas for the determinants of the matrices $M^{\lambda / \mu}$ and $\mathcal{M}^{\lambda / \mu}$, which prove Jockusch's original conjecture that $\operatorname{det} M^{\lambda / \mu}$ has only integer roots. We define a Jeu de Taquin algorithm for standard matchings, and compare this algorithm to the usual Jeu de Taquin algorithm defined by Schützenberger for standard tableaux. The formulas for the determinants of $M^{\lambda / \mu}$ and $\mathcal{M}^{\lambda / \mu}$ have elegant statements in terms of this new Jeu de Taquin algorithm.
\end{abstract}

\section{Contents}

1 Introduction $\mathbf{2}$

1.1 Acknowledgments . . . . . . . . . . . . . . . . 8

*Subject Class 05E15, 05E10

${ }^{\dagger}$ This research was supported in part by a Department of Education graduate fellowship at the University of Michigan 
2 Determinants of $M$ and $\mathcal{M} \quad 8$

2.1 Column permutations of standard matchings . . . . . . . . 8

2.2 Product formulas for $M$ and $\mathcal{M}$. . . . . . . . . . . . . . . 12

2.3 Eigenvalues of $T_{k}(x)$ and $\mathcal{T}_{k}\left(y_{1}, \ldots, y_{n}\right) \ldots \ldots \ldots$

2.4 The column span of $P$. . . . . . . . . . . . . . . . . . . 14

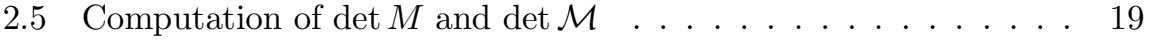

3 Jeu de Taquin for standard matchings $\quad 23$

3.1 Definition of the algorithm . . . . . . . . . . . . 23

3.2 Jeu de Taquin preserves standardness . . . . . . . . . . . . 25

3.3 Dual Knuth equivalence with JdT for tableaux . . . . . . . . . . 31

3.4 The normal shape obtained via JdT . . . . . . . . . . . . 35

3.5 An alternate statement of the main theorem . . . . . . . . . 38

\section{Introduction}

Brauer's Centralizer Algebras were introduced by Richard Brauer [Brr] in 1937 for the purpose of studying the centralizer algebras of orthogonal and symplectic groups on the tensor powers of their defining representations. An interesting problem that has been open for many years now is to determine the algebra structure of the Brauer centralizer algebras $A_{f}^{(x)}$. Some results about the semisimplicity of these algebras were found by Brauer, Brown and Weyl, and have been known for quite a long time (see [Brr],[Brn],[Wl]). More recently, Hanlon and Wales [HW1] have been able to reduce the question of the structure of $A_{f}^{(x)}$ to finding the ranks of certain matrices $Z^{\lambda / \mu}(x)$. Finding these ranks has proved very difficult in general. They have been found in several special cases, and there are many conjectures about these matrices which are supported by large amounts of computational evidence. One conjecture arising out of this work was that $A_{f}^{(x)}$ is semisimple unless $x$ is a rational integer. Wenzl [Wz] has used a different approach (involving "the tower construction" due to Vaughn Jones [Jo]) to prove this important result. In our work we take the point of view taken by Hanlon and Wales in [HW1]-[HW4], and we pay particular attention to the case where $x$ is a rational integer.

We consider subsets of $\mathbb{Z}^{+} \times \mathbb{Z}^{+}$, which we will think of as the set of positions in an infinite matrix, whose rows are numbered from top to bottom, and whose columns are numbered from left to right. Thus, the element $(i, j)$ will be thought of as the position in the $i$ th row, and $j$ th column of the matrix. These positions will be called boxes.

Definition 1.1. Define the partial order $<_{s}$, "the standard order" on $\mathbb{Z}^{+} \times \mathbb{Z}^{+}$, by $x \leq_{s} y$ if $x$ appears weakly North and weakly West of $y$.

Definition 1.2. Define the total order $<_{h}$, "the Hebrew order" on $\mathbb{Z}^{+} \times \mathbb{Z}^{+}$, by $x<_{h} y$ if $y$ is either strictly South of $x$, or if $y$ is in the same row as $x$ and strictly West of $x$ in that row. 
Definition 1.3. A finite subset $D \subset \mathbb{Z}^{+} \times \mathbb{Z}^{+}$will be called a diagram. A matching of the diagram $D$ is a fixed point free involution $\epsilon: D \rightarrow D$. A matching $\delta$ of the diagram $D$ is called standard if for every $x, y \in D, x<_{s} y$ implies that $\delta(x)<_{h} \delta(y)$.

We will usually use $\epsilon$ to denote an arbitrary matching, while $\delta$ will be reserved for standard matchings.

It will sometimes be convenient to think of matchings in a slightly different way, namely as 1-factors. A 1-factor is a graph such that every vertex is incident with exactly one edge. If $\epsilon$ is a matching of $D$, then we can think of $\epsilon$ as a 1factor by putting an edge between $x$ and $\epsilon(x)$ for all $x \in D$. Note that if there is a matching of shape $D$, then $D$ must contain an even number of boxes.

Example 1.1. There are three matchings of shape $(4,2) /(2)$. They are represented below as the 1 -factors $\delta, \delta^{\prime}$ and $\epsilon$. The matchings $\delta$ and $\delta^{\prime}$ are both standard, while $\epsilon$ is not standard.

$\delta$

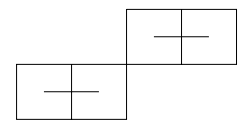

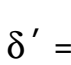

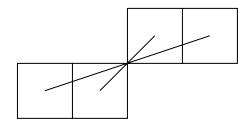

$\varepsilon=$

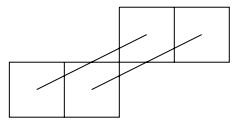

Remark 1.1. An immediate consecuence of the definition for a standard matching is that one can never have an edge between boxes $x$ and $y$ if both $x<_{s} y$ and $x<_{h} y$. This means that there can be no NW-SE edges in a standard matching, nor N-S (or vertical) edges. There can be E-W (horizontal) edges however.

Let $F_{D}$ be the set of matchings of $D$, and let $V_{D}$ be the real vector space with basis $F_{D}$. Let $A_{D}$ be the set of standard matchings of $D$.

If $\lambda / \mu$ is a skew shape then let $[\lambda / \mu] \subset \mathbb{Z}^{+} \times \mathbb{Z}^{+}$be the set of boxes $(i, j)$ such that $\mu_{i}<j \leq \lambda_{i}$. If $D=[\lambda / \mu]$ for some skew shape $\lambda / \mu$, then we will sometimes drop the brackets, especially in subscripts. For example, by convention $F_{\lambda / \mu}=F_{[\lambda / \mu]}$.

Suppose that $\lambda / \mu$ is a skew shape. Let $S_{\lambda / \mu}$ denote the symmetric group on the set $[\lambda / \mu]$. There is an $S_{\lambda / \mu}$ action on $F_{\lambda / \mu}$ given by

$$
(\pi \epsilon)(x)=\pi\left(\epsilon\left(\pi^{-1} x\right)\right)
$$

where $\pi \in S_{\lambda / \mu}$. In terms of 1-factors, this is equivalent to saying that $x$ and $y$ are adjacent in $\epsilon$ if and only if $\pi(x)$ and $\pi(y)$ are adjacent in $\pi \epsilon$.

Let $C_{\lambda / \mu}$ (resp. $R_{\lambda / \mu}$ ), the column stabilizer (resp. row stabilizer) of $[\lambda / \mu]$, be the subgroup of $S_{\lambda / \mu}$, consisting of permutations $\pi$, such that $\pi(x)$ is in the same column (resp. row) as $x$, for all $x \in[\lambda / \mu]$.

If $\epsilon_{1}$ and $\epsilon_{2}$ are matchings of shape $[\lambda / \mu]$, we obtain a new graph on the vertex set $[\lambda / \mu]$ by simply superimposing the two matchings. We denote this new graph by $\epsilon_{1} \cup \epsilon_{2}$. We define $\gamma\left(\epsilon_{1}, \epsilon_{2}\right)$ to be the number of cycles in $\epsilon_{1} \cup \epsilon_{2}$ (which is the same as the number of connected components in $\epsilon_{1} \cup \epsilon_{2}$ ).

Example 1.2. Below are two matchings of shape $(5,4,2) /(2,1), \epsilon_{1}$ and $\epsilon_{2}$. Here $\gamma\left(\epsilon_{1}, \epsilon_{2}\right)=2$. 


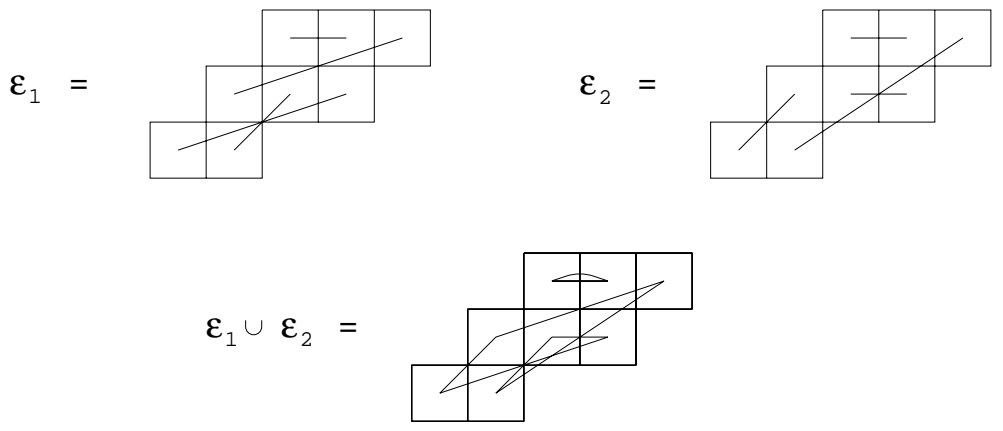

We define the $A_{\lambda / \mu} \times A_{\lambda / \mu}$ matrix $M=M^{\lambda / \mu}(x)$ as follows:

$$
M_{i j}=M_{\delta_{i}, \delta_{j}}=\sum_{\sigma \in C_{\lambda / \mu}} \sum_{\tau \in R_{\lambda / \mu}} \operatorname{sgn}(\sigma) x^{\gamma\left(\sigma \tau \delta_{i}, \delta_{j}\right)}
$$

where $A_{\lambda / \mu}=\left\{\delta_{1}, \ldots \delta_{s}\right\}$.

We have defined a matching of shape $\lambda / \mu$ to be a fixed point free involution of $[\lambda / \mu]$, or equivalently a 1-factor on $[\lambda / \mu]$. If $|\mu|=m$, and $|\lambda / \mu|=2 k$, then we can also think of a matching of shape $\lambda / \mu$ as a labelled $(m, k)$ partial 1 factor on the set $[\lambda]$. A labeled $(m, k)$ partial 1-factor is a graph on $f=m+2 k$ points, where $2 k$ points are incident with exactly one edge, and $m$ points (called free points) are incident with no edges. These free points are labelled with the numbers $1,2, \ldots, m$. For a matching of shape $\lambda / \mu$, the free points are the boxes in $[\mu]$, and we label them in order from left to right in each row, from the top row to the bottom row.

Let $P_{\lambda, m}$ be the set of labelled $(m, k)$ partial 1-factors on $[\lambda]$. There is an $S_{\lambda}$ action on $P_{\lambda, m}$ given by saying that $x$ and $y$ are adjacent in $\epsilon$, if and only if $\pi(x)$ and $\pi(y)$ are adjacent in $\pi \epsilon$, and if $x$ is a free point in $\epsilon$ with label $i$, then $\pi(x)$ is a free point in $\pi \epsilon$ with label $i$. Note that $F_{\lambda / \mu} \subseteq P_{\lambda, m}$ and that the $S_{\lambda / \mu}$ action we defined on $F_{\lambda / \mu}$ is equivalent to the restriction of the $S_{\lambda}$ action on $F_{\lambda / \mu}$ to those permutations in $S_{\lambda}$ that fix $[\mu]$ pointwise. As before, we define $R_{\lambda}$ (resp. $C_{\lambda}$ ) to be the subgroup of $S_{\lambda}$ that stabilizes the rows (resp. columns) of $\lambda$.

Suppose that $\epsilon_{1}, \epsilon_{2}$ are labelled $(m, k)$ partial 1-factors in $P_{\lambda, m}$. Then $\epsilon_{1} \cup \epsilon_{2}$ is a graph on the vertex set $[\lambda]$ consisting of exactly $m$ paths (an isolated point is considered a path of length zero), and some number $\gamma\left(\epsilon_{1}, \epsilon_{2}\right)$ of cycles, each of which has even length. Each of the $m$ paths is a path from one labelled point to another. Let $\zeta\left(\epsilon_{1}, \epsilon_{2}\right)$ equal 1 if each path has the same label at both endpoints, and 0 otherwise. We can now define $Z=Z^{\lambda / \mu}(x)$ as follows.

$$
Z_{i j}=Z_{\delta_{i}, \delta_{j}}=\sum_{\sigma \in C_{\lambda}} \sum_{\tau \in R_{\lambda}} \operatorname{sgn}(\sigma) \zeta\left(\sigma \tau \delta_{1}, \delta_{2}\right) x^{\gamma\left(\sigma \tau \delta_{i}, \delta_{j}\right)}
$$

The terms that appear in $M$ are a subset of those that appear in $Z$ because if $\sigma \in C_{\lambda / \mu}$ then $\sigma$ fixes $[\mu]$ pointwise, and the same is true for all $\tau \in R_{\lambda / \mu}$. Thus, 
$\sigma \tau$ fixes $[\mu]$ pointwise, and it follows that $\zeta\left(\sigma \tau \delta_{i}, \delta_{j}\right)=1$ for all $i, j$. One can think of $M$ as the component of $Z$ that leaves [ $\mu$ ] fixed. In this paper, we are able to find the determinant of $M$ precisely. In order to find the determinant of $Z$, one might try to get an intermediate result which would involve matrices which only allowed the boxes in $[\mu]$ to move in certain restricted ways. If one could get results about such matrices, and then find a way to remove the restrictions, one might finally arrive at the determinant of $Z$. This would be a powerful tool for finding the rank of $Z$, which is equivalent to determining the algebra structure of $A_{f}^{(x)}$ completely.

If $x=n \in \mathbb{Z}^{+}$, one can generalize the definition of the matrix $Z$ by introducing power sum symmetric functions to keep track of the lengths of cycles. Recall that for $i \geq 0$, the $i$ th power sum on $y_{1}, \ldots, y_{n}$ is given by

$$
p_{i}\left(y_{1}, \ldots, y_{n}\right)=\sum_{j=1}^{n} y_{j}^{i} .
$$

Note that $p_{i}(1, \ldots, 1)=n$ for all $i$. For any partition $\nu=\left(\nu_{1}, \nu_{2}, \ldots, \nu_{l}\right)$, we define $p_{\nu}=\prod p_{\nu_{i}}$. If $\epsilon_{1}$ and $\epsilon_{2}$ are labelled $(m, k)$ partial 1-factors in $P_{\lambda, m}$, then define $\Gamma\left(\epsilon_{1}, \epsilon_{2}\right)$ to be the partition having one part for each cycle in $\epsilon_{1} \cup \epsilon_{2}$. If a cycle in $\epsilon_{1} \cup \epsilon_{2}$ has length $2 r$, then its corresponding part in $\Gamma\left(\epsilon_{1}, \epsilon_{2}\right)$ is $r$.

Example 1.3. Below are two $(3,4)$ partial 1-factors, $\epsilon_{1}$ and $\epsilon_{2}$. In this example $\gamma\left(\epsilon_{1}, \epsilon_{2}\right)=2, \Gamma\left(\epsilon_{1}, \epsilon_{2}\right)=(2,1)$ and $\zeta\left(\epsilon_{1}, \epsilon_{2}\right)=1$.

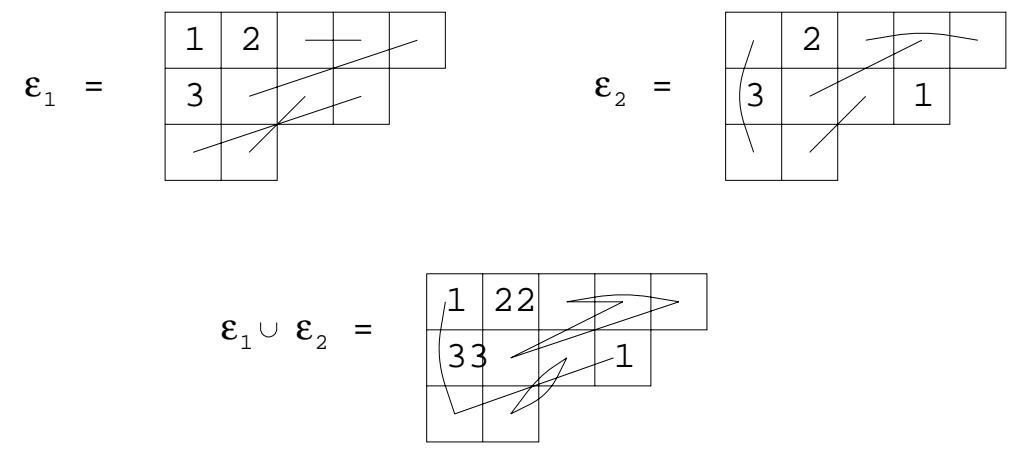

Define $\mathcal{Z}=\mathcal{Z}^{\lambda / \mu}\left(y_{1}, \ldots, y_{n}\right)$ by

$$
\mathcal{Z}_{i j}=\mathcal{Z}_{\delta_{i}, \delta_{j}}=\sum_{\sigma \in C_{\lambda}} \sum_{\tau \in R_{\lambda}} \operatorname{sgn}(\sigma) \zeta\left(\sigma \tau \delta_{1}, \delta_{2}\right) p_{\Gamma\left(\sigma \tau \delta_{i}, \delta_{j}\right)}
$$

It is clear that $\mathcal{Z}^{\lambda / \mu}(1, \ldots, 1)=Z^{\lambda / \mu}(n)$.

In [HW1], Hanlon and Wales show that if $\mu=\emptyset$ and $\lambda$ consists entirely of even parts (such a partition is called even), then $\mathcal{Z}^{\lambda / \mu}\left(y_{1}, \ldots, y_{n}\right)$ is a one by one matrix whose only entry is a scalar multiple of the zonal polynomial $z_{\nu}\left(y_{1}, \ldots, y_{n}\right)$ where $\lambda=2 \nu$ (i.e. $\lambda_{i}=2 \nu_{i}$ for all $i$ ). Zonal polynomials 
were introduced by A.T. James (see [Ja1]-[Ja3]) in connection with his work on multidimensional generalizations of the chi-squared distribution. Here we will just say that $z_{\nu}$ is a homogeneous symmetric function of degree $|\nu|$, and $z_{\nu}(1, \ldots, 1)=h_{\lambda}(n)$, where $h_{\lambda}(x)$ is the single entry of the matrix $Z^{\lambda / \emptyset}(x)$. A formula for $h_{\lambda}(x)$ is given in [HW1] which we state as Theorem 2.6.

If $|\lambda / \mu|=2 k$, then in terms of the monomials $y_{1}^{i_{1}} \cdots y_{n}^{i_{n}}$, the entries of the matrix $\mathcal{Z}$ have degree at most $k$. To see this, observe that the degree of $p_{\Gamma\left(\sigma \tau \delta_{i}, \delta_{j}\right)}$ is equal to $\left|\Gamma\left(\sigma \tau \delta_{i}, \delta_{j}\right)\right|$, and that $\left|\Gamma\left(\sigma \tau \delta_{i}, \delta_{j}\right)\right| \leq k$ because there are $2 k$ edges in $\sigma \tau \delta_{i} \cup \delta_{j}$. If all $2 k$ edges are contained in cycles then the term will have degree $k$, but if any edges are contained in paths, then the degree will be smaller than $k$.

Define $\mathcal{M}=\mathcal{M}^{\lambda / \mu}\left(y_{1}, \ldots, y_{n}\right)$ to be the matrix obtained from $\mathcal{Z}$ by extracting the terms of degree $k$. Thus, $\mathcal{M}$ will be a matrix consisting entirely of zeroes and terms of degree $k$. As we noted above, in order for a term in $\mathcal{Z}$ to have degree $k$, every edge in $\sigma \tau \delta_{i} \cup \delta_{j}$ must be contained in a cycle, or equivalently every path must be an isolated point (and this point must have the same label in $\sigma \tau \delta_{i}$ and $\delta_{j}$ ). It is not hard to see that this happens if and only if $\tau$ and $\sigma$ are both in $S_{\lambda / \mu}$, i.e. both fix $|\mu|$ pointwise. Hence

$$
\mathcal{M}_{i j}=\mathcal{M}_{\delta_{i}, \delta_{j}}=\sum_{\sigma \in C_{\lambda / \mu}} \sum_{\tau \in R_{\lambda / \mu}} \operatorname{sgn}(\sigma) p_{\Gamma\left(\sigma \tau \delta_{i}, \delta_{j}\right)} .
$$

It follows that $\mathcal{M}^{\lambda / \mu}(1, \ldots, 1)=M^{\lambda / \mu}(n)$. In this sense, the matrix $M$ can be considered the "highest degree" part of the matrix $Z$, where we are computing the "degree" of the terms using the homogeneous degree of the corresponding terms in the matrix $\mathcal{Z}$. For example, the terms $p_{2} p_{1}$ and $p_{1}^{3}$ have the same homogeneous degree three. But when we specialize $p_{2}=p_{1}=n$, the corresponding terms are $n^{2}$ and $n^{3}$ respectively. In the sense described above however, both terms have "degree" equal to three. Also, there is more than one way to obtain $n^{i}$ for any $i>1$. This means that one generally cannot reconstruct $\mathcal{M}$ by substitution in $M(n)$.

The matrix $\mathcal{M}$ has an interesting algebraic interpretation which we briefly describe here. To do this we give a short description of the algebra $A_{f}^{(x)}$ and the closely related algebra $A_{f}^{\Lambda}$. See [HW1] for a more complete description. Both algebras have the same basis, namely the set of 1 -factors on $2 f$ points. To define the product of two such 1-factors $\delta_{1}$ and $\delta_{2}$, we construct a graph $B\left(\delta_{1}, \delta_{2}\right)$. We can think of this graph as a 1 -factor $\beta\left(\delta_{1}, \delta_{2}\right)$ together with some number $\gamma\left(\delta_{1}, \delta_{2}\right)$ of cycles of even lengths $2 l_{1}, 2 l_{2}, \ldots, 2 l_{\gamma\left(\delta_{1}, \delta_{2}\right)}$. The product in $A_{f}^{(x)}$ is given by

$$
\delta_{1} * \delta_{2}=x^{\gamma\left(\delta_{1}, \delta_{2}\right)} \beta\left(\delta_{1}, \delta_{2}\right) .
$$

In $A_{f}^{\Lambda}$, the product is

$$
\delta_{1} \cdot \delta_{2}=\left(\prod_{j=1}^{\gamma\left(\delta_{1}, \delta_{2}\right)} p_{l_{j}}\left(y_{1}, \ldots, y_{n}\right)\right) \beta\left(\delta_{1}, \delta_{2}\right)
$$


where $p_{i}\left(y_{1}, \ldots, y_{n}\right)$ is the $i$ th power sum. Since $p_{i}(1, \ldots, 1)=n$ for all $i$, the specialization of $A_{f}^{\Lambda}$ to $y_{1}=\cdots=y_{n}=1$ is isomorphic to $A_{f}^{(n)}$.

In $A_{f}^{\Lambda}$ there is an important tower of ideals $A_{f}^{\Lambda}=A_{f}^{\Lambda}(0) \supset A_{f}^{\Lambda}(1) \supset A_{f}^{\Lambda}(2) \supset$ $\cdots$. Let $D_{f}^{\Lambda}(k)=A_{f}^{\Lambda}(k) / A_{f}^{\Lambda}(k+1)$. In [HW1], Hanlon and Wales express the multiplication in $D_{f}^{\Lambda}(k)$ in terms of a matrix $\mathcal{Z}_{m, k}=\mathcal{Z}_{m, k}\left(y_{1}, \ldots, y_{n}\right)$ where $f=m+2 k$. Furthermore, they show that the algebra structure of $D_{f}^{\Lambda}(k)$ for particular values of $y_{1}, \ldots, y_{n}$ is completely determined by the rank of $\mathcal{Z}_{m, k}$. Their work implies that $\operatorname{det}\left(\mathcal{Z}_{m, k}\right) \neq 0$ for the values $y_{1}, \ldots, y_{n}$ if and only if $D_{f}^{\Lambda}(k)$ is semisimple for those values.

A typical element of $D_{f}^{\Lambda}(k)$ is a sum of terms of the form $f \delta$ where $f$ is a homogeneous symmetric function and $\delta$ is a certain type of 1-factor. Let $\operatorname{gr}(f \delta)=\operatorname{deg}(f)+k$. The multiplication in $D_{f}^{\Lambda}(k)$ respects this grading in the sense that $\operatorname{gr}\left(f_{1} \delta_{1} \cdot f_{2} \delta_{2}\right) \leq \operatorname{gr}\left(f_{1} \delta_{1}\right)+\operatorname{gr}\left(f_{2} \delta_{2}\right)$. Let $\tilde{D}_{f}^{\Lambda}(k)$ be the associated graded algebra. One can construct matrices $\mathcal{M}_{m, k}=\mathcal{M}_{m, k}\left(y_{1}, \ldots, y_{n}\right)$ that play the same role in $\tilde{D}_{f}^{\Lambda}(k)$ that the $\mathcal{Z}_{m, k}$ play in $D_{f}^{\Lambda}(k)$. It turns out that $\mathcal{M}_{m, k}$ is the matrix obtained from $\mathcal{Z}_{m, k}$ by extracting highest degree terms.

Using the representation theory of the symmetric group, one can show that the matrix $\mathcal{M}_{m, k}$ is similar to a direct sum of matrices $\mathcal{M}^{\lambda / \mu}$ where $\lambda$ and $\mu$ are partitions of $f$ and $m$ respectively. These matrices $\mathcal{M}^{\lambda / \mu}$ are precisely the matrices $\mathcal{M}$ defined above. The main result of this paper is a formula for the determinant of $\mathcal{M}$, which can be interpreted as a discriminant for the algebra $\tilde{D}_{f}^{\Lambda}(k)$ in the same way that $\operatorname{det} \mathcal{Z}$ is a discriminant for $D_{f}^{\Lambda}(k)$.

This paper is split into two main sections. In section 2 , we prove several basic facts about standard matchings which are needed to compute the determinant of $M$. In particular, we find an ordering on matchings (defined in 2.4) such that if $\delta$ is a standard matching, then any column permutation of $\delta$ yields a matching which is weakly greater than $\delta$ in this order (see Theorem 2.5). In Theorem 2.11 we show that the standard matchings of shape $\lambda / \mu$ index a basis for an important vector space associated to $\lambda / \mu$. This part of the paper is very similar in flavor to standard constructions of the irreducible representations of the symmetric group. Using these two theorems, we are able to give an explict product formula for the determinant of $M$ in Theorem 2.14. This formula has the following form:

$$
\operatorname{det} M=C \prod_{2 \nu \vdash|\lambda / \mu|} h_{2 \nu}(x)^{c_{\mu 2 \nu}^{\lambda}}
$$

where $C$ is some nonzero real number, and $c_{\mu \nu}^{\lambda}$ is a Littlewood-Richardson coefficient. The same argument also shows that for $x=n \in \mathbb{Z}^{+}$,

$$
\operatorname{det} \mathcal{M}=C \prod_{2 \nu \vdash|\lambda / \mu|} z_{\nu}\left(y_{1}, \ldots, y_{n}\right)^{c_{\mu}^{\lambda} 2 \nu}
$$

where the constant $C$ is the same as the one above.

In section 3, we introduce a Jeu de Taquin algorithm for standard matchings. Much of this section is devoted to a comparison of this algorithm with 
the well known Jeu de Taquin algorithm for standard tableaux invented by Schützenberger. This makes sense because there is a natural way to think about any matching as a tableau such that a matching is standard if and only if it is standard as a tableau. In Theorem 3.3 we show that if Jeu de Taquin for matchings is applied to a standard matching of a skew shape with one box removed, then the output is another standard matching. Theorem 3.5 gives a description of how the two Jeu de Taquin algorithms compare in terms of the dual Knuth equivalence for permutations. Theorem 3.5 is used to show in Theorem 3.10 that if Jeu de Taquin is used to bring a standard matching of a skew shape to a standard matching of a normal shape (the shape of a partition), then both algorithms arrive at the same normal shape, and as a consequence of this, the standard matching of normal shape obtained from any standard matching is independent of the sequence of Jeu de Taquin moves chosen. Finally, using Theorem 3.12, a result of Dennis White [Wh] we find that the number of times the normal shape $\nu$ appears as the shape obtained from a standard matching of shape $\lambda / \mu$ using Jeu de Taquin is the Littlewood-Richardson coefficient $c_{\mu \nu}^{\lambda}$ (Theorem 3.14). Using this theorem we obtain elegant restatements of the main results from section 2 (Theorems 2.14 and 2.16) in terms of the Jeu de Taquin algorithm for standard matchings.

\subsection{Acknowledgments}

The author would like to thank William Jockusch for suggesting the problem discussed in this paper, and Phil Hanlon for valuable discussions leading to the results described here.

\section{Determinants of $M$ and $\mathcal{M}$}

\subsection{Column permutations of standard matchings}

Definition 2.1. Suppose $\epsilon$ is a matching of shape $\lambda / \mu$. Define $\frac{T(\epsilon)}{T(\epsilon)}$ to be the filling of $[\lambda / \mu]$ that puts $i$ in box $x$ if $\epsilon(x)$ is in row $i$. Define $\overline{T(\epsilon)}$ to be the filling obtained by rearranging the elements in each row of $T(\epsilon)$ in increasing order from left to right.

Example 2.1. Here are $T(\epsilon)$ and $\overline{T(\epsilon)}$ for the matching $\epsilon$ shown below.
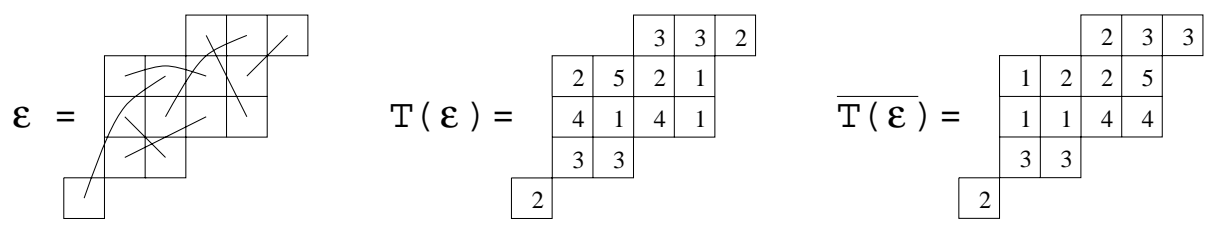

Lemma 2.1. If $\delta$ is a standard matching then $T(\delta)$ is a semistandard tableau. 
Proof. Suppose $x \in[\lambda / \mu]$. If $y \in[\lambda / \mu]$ is immediately below or to the right of $x$ then $x<_{s} y$. Hence $\delta(x)<_{h} \delta(y)$, and it follows that $\delta(y)$ must be in the same row as $\delta(x)$ or below.

If $y \in[\lambda / \mu]$ is immediately below $x$, then $\delta(y)$ cannot be in the same row as $\delta(x)$ because if that were the case, then $\delta(y)$ would have to be to the left of $\delta(x)$, i.e. $\delta(y)<_{s} \delta(x)$. But this implies that $y<_{h} x$, a contradiction.

Thus, $T(\delta)$ increases weakly in its rows, and strictly in its columns.

Remark 2.1. Lemma 2.1 implies that if $\delta$ is a standard matching, then $\overline{T(\delta)}=$ $T(\delta)$.

Also, it is not hard to see that $\delta \mapsto T(\delta)$ is a bijection between standard matchings of shape $\lambda / \mu$ and semistandard tableaux of shape $\lambda / \mu$ satisfying

1. Row $i$ has an even number of $i$ 's, and

2. Row $i$ has $k j$ 's if and only if row $j$ has $k i$ 's.

For the notation described below and the following two lemmas, let $\delta$ be some fixed standard matching of shape $\lambda / \mu$.

Notation. Let $R_{i}$ denote the $i$ th row of $[\lambda / \mu]$. If $x \in[\lambda / \mu]$ then let $\operatorname{row}(x)$ denote the row number in which $\mathrm{x}$ appears. For all $i \in \mathbb{Z}$ let $C_{i}$ denote the subset of $[\lambda / \mu]$ defined as follows:

If $i \geq 0$ then $C_{i}$ is the union of the columns of $[\lambda / \mu]$ that have an $i+1$ in the first row of $\overline{T(\delta)}=T(\delta)$.

If $i<0$ then $C_{i}$ is the union of the columns of $[\lambda / \mu]$ whose top box is in row $|i|+1=1-i$. 
Example 2.2. Below is $T(\delta)$ for a standard matching for which the sets $R_{i}$ and $C_{i}$ are shown.
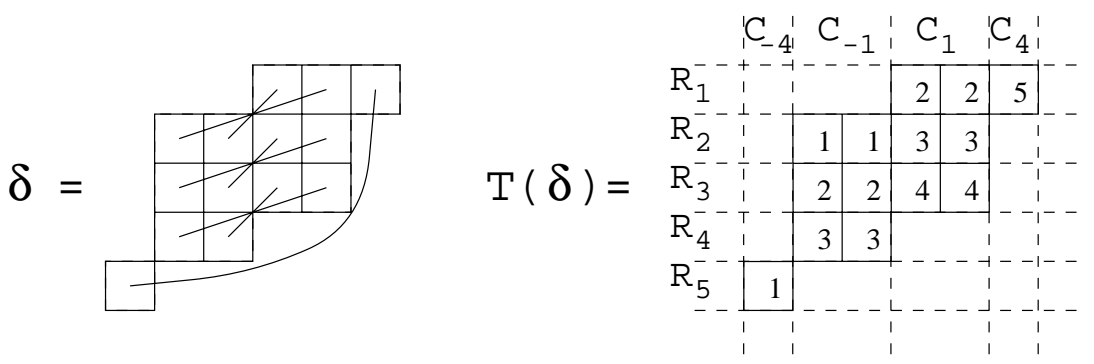

Remark 2.2. Note that $R_{i} \cap C_{j}=\emptyset$ unless $j \geq 1-i$.

Lemma 2.2. Suppose $x \in C_{i}$, and $\delta(x) \in C_{j}$. Then

a. $\quad \operatorname{row}(\delta(x)) \geq \operatorname{row}(x)+i$, and $\operatorname{row}(x) \geq \operatorname{row}(\delta(x))+j$,

b. $i+j \leq 0$, and $\delta(x)$.

c. If $i=-j$, then $\operatorname{row}(x)=\operatorname{row}(\delta(x))+i$, i.e. $x$ is exactly $i$ rows below

Proof. Suppose $x \in R_{k}$, and $\delta(x) \in R_{l}$. In $T(\delta)$, position $x$ has an $l$ in it. Lemma 2.1 implies that $l \geq k+i$, which is precisely the first statement in $a$. The second statement in $a$ follows from the first by noting that $\delta(\delta(x))=x$.

Both $b$ and $c$ are immediate consequences of $a$.

Lemma 2.3. Suppose $i \geq 0$, and $x \in C_{i}$ satisfies $\operatorname{row}(\delta(x))=\operatorname{row}(x)+i$. Suppose also that there are $k$ columns in $C_{i}$ to the right of $x$. Then there are no more than $k$ columns in $C_{-i}$ to the left of $\delta(x)$.

Proof. Let $z$ be a box in $R_{\text {row }(\delta(x))} \cap C_{-i}$ that lies to the left of $\delta(x)$. We have $z<_{s} \delta(x)$, so $\delta(z)<_{h} x$. Thus, $\delta(z)$ lies in $R_{\text {row }(x)}$ or above. But Lemma $2.2 a$ implies that

$$
\operatorname{row}(\delta(z)) \geq \operatorname{row}(z)-i=\operatorname{row}(x)
$$

i.e. $\delta(z)$ lies in $R_{\text {row }(x)}$ or below. Furthermore, Lemma $2.2 b$ says that $\delta(z)$ lies in $C_{i}$ or to its left. Therefore, $\delta(z)$ lies in $R_{\text {row }(x)} \cap C_{i}$ and to the right of $x$. The number of such boxes $z$ is obviously bounded by $k$.

One useful consequence of these two lemmas is the following Corollary.

Corollary 2.4. Suppose $\lambda \vdash 2 k$. Then, if $\lambda$ is even, there is exactly one standard matching of shape $\lambda$. If $\lambda$ is not even, then there are no standard matchings of shape $\lambda$. 
Proof. Suppose that $\delta$ is a standard matching of shape $\lambda$. Observe that because $\lambda$ is normal, $C_{i}=\emptyset$ for all $i<0$. But now, Lemma $2.2 b$ implies that $C_{i}=\emptyset$ for all $i>0$ as well. Thus $[\lambda]=C_{0}$.

Now, by Lemma $2.2 c$, all edges of $\delta$ must be horizontal, which implies immediately that $\lambda$ is even. Furthermore, for a single row of an even number of boxes, it is not hard to see that there is exactly one matching that is standard, namely the one that connects the $i$ th box from the left to the $i$ th box from the right of the row. Putting all of these rows together, we see that the resulting matching is indeed standard.

Definition 2.2. Define the total order $\prec$ on tableaux of shape $\lambda / \mu$ with weakly increasing rows as follows:

Write $S \prec T$, if the word $w_{S}$ obtained by reading the rows of $S$ from left to right, from the top row to the bottom row, lexicographically precedes the corresponding word $w_{T}$, obtained by reading $T$ in the same order.

Remark 2.3. Note that $\prec$ induces a total order on standard matchings (but not all matchings) of shape $\lambda / \mu$, via $\delta_{1} \prec \delta_{2}$ if and only if $\overline{T\left(\delta_{1}\right)} \prec \overline{T\left(\delta_{2}\right)}$. This follows from Remark 2.1, which implies that a standard matching $\delta$ is completely determined by $\overline{T(\delta)}$. Note that this is not true of matchings in general. In other words, there could be several matchings with the same associated tableau, but there can be at most one standard matching associated to any tableau.

Theorem 2.5. Suppose $\sigma \in C_{\lambda / \mu}$, and suppose $\delta$ is a standard matching of shape $\lambda / \mu$. Then $\overline{T(\sigma \delta)} \succeq \overline{T(\delta)}$, and $\overline{T(\sigma \delta)}=\overline{T(\delta)}$ if and only if $\sigma \delta=\delta$.

Proof. We induct on $|[\lambda / \mu]|$. If $|[\lambda / \mu]|=2$, the statement of the theorem is trivially true since there is only one matching of shape $\lambda / \mu$ in that case.

If $|[\lambda / \mu]|>2$, then let $k$ be the smallest nonnegative integer such that $C_{k} \neq \emptyset$, i.e. $k+1$ is the entry of $\overline{T(\delta)}$ in the leftmost box of the first row. Let $H$ be the subgroup of the column stabilizer of $[\lambda / \mu]$ consisting of permutations $\tau$ such that $(\tau \delta)(x)=\delta(x)$ for all $x \in R_{1} \cap C_{k}$.

Let $A=R_{1} \cap C_{k}$, and let $\alpha$ (resp. $\beta$ ) be the word obtained by reading the entries corresponding to the positions of $A$ in $\overline{T(\delta)}$ (resp. $\overline{T(\sigma \delta)}$ ) from left to right. Note that $\alpha$ is a word consisting entirely of $k+1$ 's.

First, we will show that $\beta$ cannot precede $\alpha$ in the lexicographic order. Then, we will show that if $\beta=\alpha$, then $\sigma \in H$. Once we have shown these two things, we will be able to finish the proof of the theorem by induction.

To show that $\beta$ does not precede $\alpha$ lexicographically, we show that $\beta$ cannot contain any letters smaller than $k+1$. If $\beta$ contained $l<k+1$, then for some $x \in R_{1},(\sigma \delta)(x) \in R_{l}$. Suppose $x \in C_{i}$. Note that $i \geq k$, and $(\sigma \delta)(x) \in C_{j}$ for some $j \geq 1-l>-k$. (Recall that $R_{l} \cap C_{j}=\emptyset$ unless $j \geq 1-l$ ). Since $\sigma$ stabilizes the columns of $[\lambda / \mu]$, this implies that there exists some $z \in C_{i}$ such that $\delta(z) \in C_{j}$. But $i+j>k-k=0$, which contradicts Lemma 2.2 $b$. We conclude that $\beta$ cannot precede $\alpha$ lexicographically. Observe that this same argument shows that if $x \in R_{1}$ and $(\sigma \delta)(x) \in R_{k+1}$, then $x \in C_{k}$, and $(\sigma \delta)(x) \in C_{-k}$. 
Suppose now that $\beta=\alpha$, and suppose that $\sigma \notin H$. Write the elements of $A$ as $x_{1}, x_{2}, \ldots, x_{a}$ in order from right to left, and let $x_{i}$ be the rightmost box in $A$ such that $y=(\sigma \delta)\left(x_{i}\right) \neq \delta\left(x_{i}\right)$. In $R_{k+1}$, let $y_{1}, y_{2}, \ldots, y_{a}$ be the first $a$ boxes from left to right. Note that for all $j \in\{1, \ldots, a\}, \delta\left(x_{j}\right)=y_{j}$. Furthermore, for each $j \in\{1, \ldots, a\}, \delta\left(y_{j}\right)=x_{j} \in R_{1}$, and hence $\overline{T(\delta)}$ has a 1 in position $y_{j}$ for all such $j$. This implies that each such $y_{j}$ must be at the top of its column, and hence in $C_{-k}$.

Since $\beta=\alpha, y \in R_{k+1}$. Also, $y \neq y_{j}$ for any $j \leq i$. Thus $y$ is farther right in $R_{k+1}$ than $y_{i}$. Since $\sigma$ stabilizes the columns of $[\lambda / \mu]$, this implies that there is some $z \in C_{k}$ in the same column as $x_{i}$, such that $\delta(z)$ is in the same column as $y$. Now, by the comments above, $\delta(z)$ must be in $C_{-k}$ or to its right. Lemma $2.2 b$ implies that $\delta(z)$ must be in $C_{-k}$ or to its left. Thus $\delta(z) \in C_{-k}$, and now Lemma $2.2 c$ implies that $\delta(z)$ is exactly $k$ rows below $z$. By Lemma 2.3, $\delta(z)$ must be in the same column as $y_{i}$, or to its left. But this contradicts the fact that $y$ lies to the right of $y_{i}$ and $\delta(z)$ is in the same column as $y$. It follows that $\sigma \in H$ as desired.

We have shown that

$$
\sigma \notin H \Longrightarrow \overline{T(\sigma \delta)} \succ \overline{T(\delta)}
$$

If $\sigma \in H$ then let $\mu^{*}$ be the partition such that $\left[\mu^{*}\right]=[\mu] \cup A \cup \delta(A)$. Let $\delta^{*}$ be the standard matching of shape $\lambda / \mu^{*}$ that is $\delta$ restricted to $\left[\lambda / \mu^{*}\right]$. Let $\sigma^{*} \in H$ be a column permutation of $[\lambda / \mu]$ such that $\sigma^{*}$ fixes $A \cup \delta(A)$ pointwise, and $\sigma^{*} \delta=\sigma \delta$. It is clear that such a $\sigma^{*}$ exists because of the definition of $H$. We can think of $\sigma^{*}$ as a column permutation of $\left[\lambda / \mu^{*}\right]$.

By induction, we have

$$
\overline{T\left(\sigma^{*} \delta^{*}\right)} \succeq \overline{T\left(\delta^{*}\right)}
$$

and

$$
\overline{T\left(\sigma^{*} \delta^{*}\right)}=\overline{T\left(\delta^{*}\right)} \Longleftrightarrow \sigma^{*} \delta^{*}=\delta^{*} .
$$

Now, $\overline{T(\sigma \delta)}=\overline{T\left(\sigma^{*} \delta\right)}$ has the same letters as $\overline{T(\delta)}$ in the positions in $A \cup \delta(A)$, so it is clear from (2.3) that $T(\sigma \delta) \succeq T(\delta)$. Moreover, (2.4) implies that $T(\sigma \delta)=$ $T(\delta)$ if and only if $\sigma \delta=\delta$.

\subsection{Product formulas for $M$ and $\mathcal{M}$}

For the remainder of this paper, we assume that $|\lambda / \mu|=2 k$. It will be convenient to think of the matrices $M$ and $\mathcal{M}$ as products of three matrices,

$$
\begin{aligned}
M & =P^{t} T_{k}(x) J \\
\mathcal{M} & =P^{t} \mathcal{T}_{k}\left(y_{1}, \ldots, y_{n}\right) J .
\end{aligned}
$$

Here, $P$ is the $F_{\lambda / \mu} \times A_{\lambda / \mu}$ matrix with $(\epsilon, \delta)$ entry equal to the coefficient of $\epsilon$ in $e(\delta) \in V_{\lambda / \mu}$, where $e \in \mathbb{R}\left[S_{\lambda / \mu}\right]$ is given by

$$
e=\sum_{\sigma \in C_{\lambda / \mu}} \sum_{\tau \in R_{\lambda / \mu}} \operatorname{sgn}(\sigma) \sigma \tau .
$$


In other words, $P$ is the matrix whose $i$ th column is the expansion of $e\left(\delta_{i}\right)$ in terms of the basis $F_{\lambda / \mu}$, where $\delta_{i}$ is the $i$ th standard matching of shape $\lambda / \mu$.

The matrix $T_{k}(x)$ is the $F_{\lambda / \mu} \times F_{\lambda / \mu}$ matrix with $\left(\epsilon_{1}, \epsilon_{2}\right)$ entry given by

$$
T_{k}(x)_{\epsilon_{1}, \epsilon_{2}}=x^{\gamma\left(\epsilon_{1}, \epsilon_{2}\right)} .
$$

We define $\mathcal{T}_{k}\left(y_{1}, \ldots, y_{n}\right)$ by

$$
\mathcal{T}_{k}\left(y_{1}, \ldots, y_{n}\right)_{\epsilon_{1}, \epsilon_{2}}=p_{\Gamma\left(\epsilon_{1}, \epsilon_{2}\right)} .
$$

Note that $T_{k}(x)$ and $\mathcal{T}_{k}\left(y_{1}, \ldots, y_{n}\right)$ are symmetric matrices.

The $F_{\lambda / \mu} \times A_{\lambda / \mu}$ matrix $J$ is defined as follows:

$$
J_{\epsilon, \delta}= \begin{cases}1 & \epsilon=\delta \\ 0 & \epsilon \neq \delta\end{cases}
$$

We want to show that the $\left(\delta_{i}, \delta_{j}\right)$ entry of $P^{t} T_{k}(x) J$ is equal to the $\left(\delta_{i}, \delta_{j}\right)$ entry of $M$. If $Y$ is any matrix, then let $Y_{\epsilon}$ denote the column of $Y$ indexed by $\epsilon$. We have

$$
\begin{aligned}
\left(P^{t} T_{k}(x) J\right)_{\delta_{i}, \delta_{j}} & =P_{\delta_{i}}^{t} T_{k}(x) J_{\delta_{j}} \\
& =P_{\delta_{i}}^{t} T_{k}(x)_{\delta_{j}} \\
& =\sum_{\sigma \in C_{\lambda / \mu}} \sum_{\tau \in R_{\lambda / \mu}} \operatorname{sgn}(\sigma) T_{k}(x)_{\sigma \tau \delta_{i}, \delta_{j}} \\
& =\sum_{\sigma \in C_{\lambda / \mu}} \sum_{\tau \in R_{\lambda / \mu}} \operatorname{sgn}(\sigma) x^{\gamma\left(\sigma \tau \delta_{i}, \delta_{j}\right)} \\
& =M_{\delta_{i}, \delta_{j}} .
\end{aligned}
$$

Exactly the same argument shows that $\mathcal{M}=P^{t} \mathcal{T}_{k}\left(y_{1}, \ldots, y_{n}\right) J$ as well.

\subsection{Eigenvalues of $T_{k}(x)$ and $\mathcal{T}_{k}\left(y_{1}, \ldots, y_{n}\right)$}

Using the product formulas (2.5) for $M$ and $\mathcal{M}$, we can find product formulas for $\operatorname{det} M$ and $\operatorname{det} \mathcal{M}$. We can do this because the matrices $T_{k}(x)$ and $\mathcal{T}_{k}\left(y_{1}, \ldots, y_{n}\right)$ are known to have certain nice properties. What follows is a brief discussion of these properties. For a more detailed discussion, see [HW1].

Recall that there is an $S_{\lambda / \mu}$ action on $F_{\lambda / \mu}$ given by

$$
(\sigma \epsilon)(x)=\sigma\left(\epsilon\left(\sigma^{-1} x\right)\right) .
$$

This action can be linearly extended to $V_{\lambda / \mu}$, which defines a representation $\rho: S_{\lambda / \mu} \rightarrow \operatorname{End}\left(V_{\lambda / \mu}\right)$ as follows:

$$
(\rho(\sigma))(\epsilon)=\sigma \epsilon .
$$

The key fact about $T_{k}(x)$ and $\mathcal{T}_{k}\left(y_{1}, \ldots, y_{n}\right)$ that we need is that both commute with the action of $S_{\lambda / \mu}$. In other words, $T_{k}(x)$ and $\mathcal{T}_{k}\left(y_{1}, \ldots, y_{n}\right)$ can 
be regarded as endomorphisms of $V_{\lambda / \mu}$ satisfying the following equations for all $\sigma \in S_{\lambda / \mu}$ :

$$
\begin{aligned}
\rho(\sigma) T_{k}(x) & =T_{k}(x) \rho(\sigma) \\
\rho(\sigma) \mathcal{T}_{k}\left(y_{1}, \ldots, y_{n}\right) & =\mathcal{T}_{k}\left(y_{1}, \ldots, y_{n}\right) \rho(\sigma)
\end{aligned}
$$

This is an easy consequence of the following easy to prove identities (see [HW1]).

$$
\begin{aligned}
\gamma\left(\sigma \epsilon_{1}, \sigma \epsilon_{2}\right) & =\gamma\left(\epsilon_{1}, \epsilon_{2}\right) \\
\Gamma\left(\sigma \epsilon_{1}, \sigma \epsilon_{2}\right) & =\Gamma\left(\epsilon_{1}, \epsilon_{2}\right)
\end{aligned}
$$

Furthermore, the $S_{\lambda / \mu}$ action on $F_{\lambda / \mu}$ is equivalent to the action on the conjugacy class of fixed point free involutions of $S_{\lambda / \mu}$. It follows from ([M] Ex.5, p.45) that

$$
V_{\lambda / \mu}=\bigoplus_{2 \nu} V_{2 \nu}
$$

where the sum runs over all even partitions $2 \nu \vdash 2 k$. Here, $V_{2 \nu}$ denotes a submodule of $V_{\lambda / \mu}$, isomorphic to the irreducible $S_{\lambda / \mu}$ module indexed by $2 \nu$. In this notation, $V_{(2 k)}$ is isomorphic to the trivial representation, while $V_{\left(1^{2 k}\right)}$ is isomorphic to the sign representation.

Since $V_{\lambda / \mu}$ decomposes into a direct sum of irreducibles $V_{2 \nu}$, each of which has multiplicity 1, it follows from Schur's Lemma that $T_{k}(x)$ restricted to $V_{2 \nu}$ is a scalar operator denoted $h_{2 \nu}(x) I$. Similarly, $\mathcal{T}_{k}\left(y_{1}, \ldots, y_{n}\right)$ restricted to $V_{2 \nu}$ is a scalar operator. Hanlon and Wales [HW1] compute both of these scalars.

Theorem 2.6. [HW1] Let $2 \nu \vdash 2 k$. Then

$$
h_{2 \nu}(x)=\prod_{(i, 2 j) \in[2 \nu]}(x+2 j-i-1) .
$$

Theorem 2.7. [HW1] Let $2 \nu \vdash 2 k$. Then $\mathcal{T}_{k}\left(y_{1}, \ldots, y_{n}\right)$ restricted to $V_{2 \nu}$ is the scalar operator $z_{\nu}\left(y_{1}, \ldots, y_{n}\right) I$, where $z_{\nu}\left(y_{1}, \ldots, y_{n}\right)$ is the zonal polynomial indexed by $\nu$.

\subsection{The column span of $P$}

In order to analyze $M$ and $\mathcal{M}$, we will consider how $T_{k}(x)$ and $\mathcal{T}_{k}\left(y_{1}, \ldots, y_{n}\right)$ act on the column span of $P$. It turns out that this span is the same as the space $e\left(V_{\lambda / \mu}\right)=\left\langle e(\epsilon): \epsilon \in F_{\lambda / \mu}\right\rangle$, where $e$ is defined in equation (2.6). We will show, in fact, that the columns of $P$ form a basis for this space.

Definition 2.3. We say that a matching $\epsilon$ of shape $\lambda / \mu$ is row (column) increasing if every pair $x<_{s} y \in[\lambda / \mu]$, that lie in the same row (column), satisfies $\epsilon(x)<_{h} \epsilon(y)$.

Remark 2.4. By Lemma 3.1 A matching $\delta$ of shape $\lambda / \mu$ is standard if and only if it is both row and column increasing. 
Lemma 2.8. For any matching $\epsilon \in F_{\lambda / \mu}$, there exists a row permutation $\tau \in$ $R_{\lambda / \mu}$, such that $\tau \epsilon$ is row increasing.

Proof. For this proof we use the description of a matching as a 1-factor. For every $i$, we can choose a permutation $\pi^{(i)}$ which permutes row $i$ as follows. All the boxes in row $i$ that are attached to row 1 are moved to the far left of row $i$. The boxes that are attached to row 2 are moved to the right of those attached to row 1 but to the left of every other box, and so on. So in $\pi^{(i)} \epsilon$, if $x$ is to the left of $y$ in row $i$, then

$$
\operatorname{row}\left(\pi^{(i)} \epsilon(x)\right) \leq \operatorname{row}\left(\pi^{(i)} \epsilon(y)\right) .
$$

If $j \neq i$, then $\pi^{(i)}$ has no effect on the row to which any box in row $j$ is attached. Hence, if we let

$$
\pi=\pi^{(1)} \pi^{(2)} \ldots,
$$

then $\pi \epsilon$ is a matching such that if $x<_{s} y$ are in the same row then

$$
\operatorname{row}(\pi \epsilon(x)) \leq \operatorname{row}(\pi \epsilon(y)) .
$$

Let $D_{i j}$ be the set of boxes in row $i$ that are adjacent to a box in row $j$ in $\pi \epsilon$. Note that $\epsilon\left(D_{i j}\right)=D_{j i}$. Equation 2.18 implies that $D_{i j}$ and $D_{j i}$ are sets of consecutive boxes in their respective rows. We can assume that $i \leq j$ here, and choose a permutation $\sigma^{(i j)}$, of $D_{i j}$ such that the $k$ th box from the left of $D_{i j}$ is attached to the $k$ th box from the right of $D_{j i}$ in $\sigma^{(i j)} \pi \epsilon$. It is clear that $\sigma^{(i j)} \pi \epsilon$ restricted to $D_{i j} \cup D_{j i}$ is row increasing.

Furthermore, $\sigma^{(i j)}$ has no effect on $[\lambda / \mu] \backslash\left(D_{i j} \cup D_{j i}\right)$, so if we let

$$
\sigma=\prod_{i \leq j} \sigma^{(i j)}
$$

then $\sigma \pi \epsilon$ is row increasing everywhere. Therefore, $\tau=\sigma \pi$ is the desired permutation.

Now, if $\tau \in R_{\lambda / \mu}$, it is clear from the definition of $e$ in (2.6) that $e(\tau \epsilon)=e(\epsilon)$. This fact, together with Lemma 2.8 implies that we can reduce our spanning set for $e\left(V_{\lambda / \mu}\right)$ as follows

$$
e\left(V_{\lambda / \mu}\right)=\left\langle e(\epsilon): \epsilon \in F_{\lambda / \mu}, \epsilon \text { row increasing }\right\rangle
$$

Suppose that the matching $\epsilon$ of shape $\lambda / \mu$ is row increasing, but not column increasing. Then there is a box $x$ lying immediately above a box $y$ such that $\epsilon(x)>_{h} \epsilon(y)$. Let $A$ be the set of boxes in $[\lambda / \mu]$ that are in the same row as $y$, and at least as far West as $y$. Let $B$ be the set of boxes in $[\lambda / \mu]$ that are in the same row as $x$, and at least as far East as $x$.

Let $\operatorname{Sym}(A \cup B)$ denote the symmetric group on the set $A \cup B$. It is a well known fact that the following relation, called the Garnir relation, holds in the group algebra $\mathbb{R} S_{\lambda / \mu}$. 


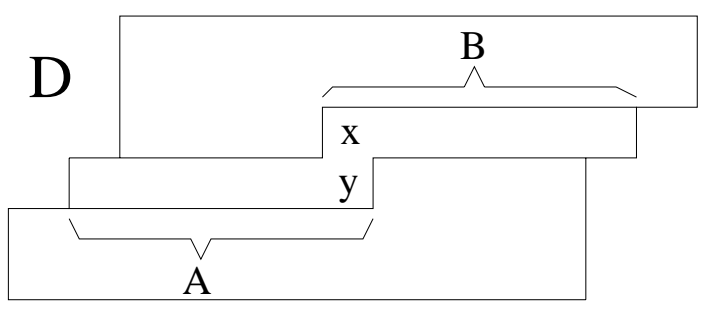

Theorem 2.9. (Garnir relation) Let $\lambda / \mu$ be a skew shape with $A, B \subset[\lambda / \mu]$ defined as above. Then

$$
\sum_{\pi \in \operatorname{Sym}(A \cup B)} e \pi=0
$$

It is useful to observe here that a row increasing matching $\epsilon$ is completely determined by $T(\epsilon)$ (see Definition 2.1). Define $\epsilon_{i, j}$ to be the number of boxes in row $i$ that are attached to a box in row $j$ in $\epsilon$. Since $\epsilon$ is row increasing, the boxes in row $i$ that are attached to row $j$ occur together in a block. Thus, it is not hard to see that a row increasing matching $\epsilon$, is uniquely determined by the numbers $\epsilon_{i, j}$, or equivalently by $T(\epsilon)$ because $\epsilon_{i, j}$ is also equal to the number of $j$ 's in row $i$ of $T(\epsilon)$. Note that for all $i$ and $j$ we have $\epsilon_{i, j}=\epsilon_{j, i}$.

Lemma 2.10. Suppose that the matching $\epsilon$ of shape $\lambda / \mu$ is row increasing, and that $x$ lies immediately above $y$ in $[\lambda / \mu]$ with $\epsilon(x)>_{h} \epsilon(y)$. Define the subsets $A, B \subset[\lambda / \mu]$ as above. If $\pi \in \operatorname{Sym}(A \cup B)$, let $\overline{\pi \epsilon}$ be the row increasing matching obtained from $\pi \epsilon$ using Lemma 2.8. Then $T(\overline{\pi \epsilon}) \preceq T(\epsilon)$.

Proof. By the definitions of $A$ and $B$, if $a \in A$ and $b \in B$, then $\epsilon(a)<_{h} \epsilon(b)$.

Suppose that $x$ is in row $i$ (so $y$ is in row $i+1$ ), and suppose that the first difference between $w_{T(\epsilon)}$ and $w_{T(\overline{\pi \epsilon})}$ occurs in row $j$.

If $j<i$, then the only boxes in row $j$ that can be affected by $\pi$ are those that are attached in $\epsilon$ to boxes in rows $i$ and $i+1$. Thus, for all $k \neq i, i+1$, we have $(\overline{\pi \epsilon})_{j, k}=\epsilon_{j, k}$. It follows that

$$
(\overline{\pi \epsilon})_{j, i}+(\overline{\pi \epsilon})_{j, i+1}=\epsilon_{j, i}+\epsilon_{j, i+1} .
$$

Since the two words differ in row $j$, we must have $(\overline{\pi \epsilon})_{j, i} \neq \epsilon_{j, i}$. If

$$
(\overline{\pi \epsilon})_{j, i}<\epsilon_{j, i}
$$

then there must be at least one box in row $j$ that is attached in $\epsilon$ to $B$. Obviously, this means that there is a box in $B$ that is attached to row $j$. The observation in the first paragraph of the proof implies that in $\epsilon$, the boxes in $A$ must be attached to row $j$ or above. If $(2.22)$ holds, then by $(2.21)$ we have $(\overline{\pi \epsilon})_{j, i+1}>\epsilon_{j, i+1}$, which implies

$$
(\overline{\pi \epsilon})_{i+1, j}>\epsilon_{i+1, j}
$$


This in turn implies that for some $k<j$, the number of boxes in row $i+1$ that are attached to row $k$ must be smaller in $\overline{\pi \epsilon}$ than in $\epsilon$, i.e.

$$
(\overline{\pi \epsilon})_{k, i+1}=(\overline{\pi \epsilon})_{i+1, k}<\epsilon_{i+1, k}=\epsilon_{k, i+1},
$$

which contradicts the assumption that $j$ is the first row in which $w_{T(\epsilon)}$ and $w_{T(\overline{\pi \epsilon})}$ differ. We conclude that

$$
(\overline{\pi \epsilon})_{j, i}>\epsilon_{j, i}
$$

which implies that $T(\overline{\pi \epsilon}) \prec T(\epsilon)$.

If $j=i$, then we know that for all $k<i$, we have

$$
(\overline{\pi \epsilon})_{i, k}=(\overline{\pi \epsilon})_{k, i}=\epsilon_{k, i}=\epsilon_{i, k},
$$

and

$$
(\overline{\pi \epsilon})_{i+1, k}=(\overline{\pi \epsilon})_{k, i+1}=\epsilon_{k, i+1}=\epsilon_{i+1, k}
$$

If

$$
(\overline{\pi \epsilon})_{i, i}<\epsilon_{i, i}
$$

then there must be at least one box in $B$ which is attached in $\epsilon$ to another box in row $i$. Hence, by the observation in the first paragraph, every box in $A$ must be attached in $\epsilon$ to row $i$ or above. So, for all $k>i+1$, the only boxes in $A \cup B$ that are attached in $\epsilon$ to row $k$ are in $B$. It is easy to see that for all $k>i+1$

$$
(\overline{\pi \epsilon})_{i, k} \leq \epsilon_{i, k}
$$

It follows that

$$
(\overline{\pi \epsilon})_{i, i}+(\overline{\pi \epsilon})_{i, i+1} \geq \epsilon_{i, i}+\epsilon_{i, i+1} .
$$

If (2.28) holds, then by (2.30) we must have

$$
(\overline{\pi \epsilon})_{i+1, i}=(\overline{\pi \epsilon})_{i, i+1}>\epsilon_{i, i+1}=\epsilon_{i+1, i} .
$$

This implies that for some $k<i$, the number of boxes in row $i+1$ that are attached to row $k$ must be smaller in $\overline{\pi \epsilon}$ than in $\epsilon$, i.e.

$$
(\overline{\pi \epsilon})_{k, i+1}=(\overline{\pi \epsilon})_{i+1, k}<\epsilon_{i+1, k}=\epsilon_{k, i+1},
$$

which contradicts the assumption that $i$ is the first row in which $w_{T(\epsilon)}$ and $w_{T(\overline{\pi \epsilon})}$ differ. We conclude that

$$
(\overline{\pi \epsilon})_{i, i} \geq \epsilon_{i, i} .
$$

If $(\overline{\pi \epsilon})_{i, i}>\epsilon_{i, i}$, then $T(\overline{\pi \epsilon}) \prec T(\epsilon)$, so we can assume that $(\overline{\pi \epsilon})_{i, i}=\epsilon_{i, i}$. In this case, by $(2.30)$ we know that $(\overline{\pi \epsilon})_{i, i+1} \geq \epsilon_{i, i+1}$. If $(\overline{\pi \epsilon})_{i, i+1}>\epsilon_{i, i+1}$, then 
$T(\overline{\pi \epsilon}) \prec T(\epsilon)$, so we can assume that $(\overline{\pi \epsilon})_{i, i+1}=\epsilon_{i, i+1}$. But now, (2.29) implies that $(\overline{\pi \epsilon})_{i, k}=\epsilon_{i, k}$ for all $k>i+1$. This means that $w_{T(\epsilon)}$ and $w_{T(\overline{\pi \epsilon})}$ are the same through row $i$, i.e. $j>i$.

If $j=i+1$, then for all $k \leq i$ we have

$$
(\overline{\pi \epsilon})_{i+1, k}=\epsilon_{i+1, k}
$$

Furthermore, for all $k>i+1$, the number of boxes in $A \cup B$ that are attached to row $k$ is the same in both $\overline{\pi \epsilon}$ and $\epsilon$. By assumption, the number of boxes in row $i$ that are attached to row $k$ is the same in $\overline{\pi \epsilon}$ and $\epsilon$, so the same is true in row $i+1$. It follows that $(\overline{\pi \epsilon})_{i+1, i+1}=\epsilon_{i+1, i+1}$ as well, which means that $w_{T(\epsilon)}$ and $w_{T(\overline{\pi \epsilon})}$ are the same through row $i+1$, contradicting our assumption.

Similarly, we can show that $j$ cannot be greater than $i+1$, because in that case we have

$$
(\overline{\pi \epsilon})_{k, l}=\epsilon_{k, l}
$$

whenever either $k \leq i+1$, or $l \leq i+1$. If $k>i+1$ and $l>i+1$, then (2.35) holds as well since $\pi$ has no effect on edges between rows $k$ and $l$ in that case. We conclude that if there is a difference between $w_{T(\epsilon)}$ and $w_{T(\overline{\pi \epsilon})}$, then it must occur in row $i$ or above, and we have already shown that the lemma holds in that case.

Theorem 2.11. The set $\left\{e(\delta): \delta \in A_{\lambda / \mu}\right\}$ forms a basis for the vector space $e\left(V_{\lambda / \mu}\right)$.

Proof. We already know that the set of $e(\epsilon)$ such that $\epsilon$ is a row increasing matching of shape $\lambda / \mu$ spans $e\left(V_{\lambda / \mu}\right)$ (see (2.20)). We can improve this slightly as follows:

$$
e\left(V_{\lambda / \mu}\right)=\left\langle e(\epsilon): \epsilon \in F_{\lambda / \mu}, \epsilon \text { row increasing, } e(\epsilon) \neq 0\right\rangle
$$

Define $W_{\lambda / \mu}=\left\langle e(\delta): \delta \in A_{\lambda / \mu}\right\rangle$. We will induct on the order $\prec$, to show that $e(\epsilon) \in W_{\lambda / \mu}$ for all row increasing matchings $\epsilon$ of shape $\lambda / \mu$. If $e(\epsilon)=0$ for all such matchings, then this is trivially true. If not, let $\epsilon_{0}$ be the row increasing matching that minimizes $T\left(\epsilon_{0}\right)$ with respect to $\prec$ among the row increasing matchings $\epsilon$ satisfying $e(\epsilon) \neq 0$.

We will show that $\epsilon_{0}$ is a standard matching of shape $\lambda / \mu$. If not, then there is some box $x$ and a box $y$ immediately below $x$ with $\epsilon_{0}(x)>_{h} \epsilon_{0}(y)$. Define the sets $A$ and $B$ as in Lemma 2.10. Let $C$ be the number of permutations $\pi \in \operatorname{Sym}(A \cup B)$ such that $\overline{\pi \epsilon_{0}}=\epsilon_{0}$. Note that $C \neq 0$, since the identity clearly works. Furthermore, note that for any matching $\epsilon$ we have

$$
e(\bar{\epsilon})=e(\epsilon)
$$

This follows from the definition of $e$, and the fact that $\bar{\epsilon}$ differs from $\epsilon$ by a row permutation. 
THE ELECTRONIC JOURNAL OF COMBINATORICS 2 (1995),\#R23

Using the Garnir relation (Theorem 2.9) we now obtain

$$
\begin{aligned}
C e\left(\epsilon_{0}\right) & =-\sum_{\pi \in \operatorname{Sym}(A \cup B), \overline{\pi \epsilon_{0}} \neq \epsilon_{0}} e\left(\pi \epsilon_{0}\right) \\
& =-\sum_{\pi \in \operatorname{Sym}(A \cup B), \overline{\pi \epsilon_{0}} \neq \epsilon_{0}} e\left(\overline{\pi \epsilon_{0}}\right) .
\end{aligned}
$$

By Lemma 2.10, each $\overline{\pi \epsilon_{0}}$ that appears in the right hand side of (2.38) satisfies $T\left(\overline{\pi \epsilon_{0}}\right) \prec T\left(\epsilon_{0}\right)$, so by our choice of $\epsilon_{0}$, we have $e\left(\overline{\pi \epsilon_{0}}\right)=0$. Hence,

$$
e\left(\epsilon_{0}\right)=0,
$$

which contradicts our choice of $\epsilon_{0}$. We deduce that there can be no such boxes $x$ and $y$, and therefore $\epsilon_{0}$ must be a standard matching, i.e. $\epsilon_{0} \in A_{\lambda / \mu}$, and $e\left(\epsilon_{0}\right) \in W_{\lambda / \mu}$.

Now, if $\epsilon$ is a row increasing matching with $T(\epsilon) \succ T\left(\epsilon_{0}\right)$, such that $\epsilon \notin A_{\lambda / \mu}$, then there is some box $x$ immediately above a box $y$ with $\epsilon(x)>_{h} \epsilon(y)$. Define $A$ and $B$ as before, and let $C$ be the number of permutations $\pi \in \operatorname{Sym}(A \cup B)$ such that $\overline{\pi \epsilon}=\epsilon$. Note that $C \neq 0$. Using the Garnir relation we obtain

$$
C e(\epsilon)=-\sum_{\pi \in \operatorname{Sym}(A \cup B), \overline{\pi \epsilon} \neq \epsilon} e(\pi \epsilon) .
$$

By Lemma 2.10, every $\overline{\pi \epsilon}$ that appears in the right hand side of (2.40) satisfies $T(\overline{\pi \epsilon}) \prec T(\epsilon)$, so by induction every term on the right hand side is in $W_{\lambda / \mu}$, which implies that $e(\epsilon) \in W_{\lambda / \mu}$ as well.

We have shown the $e\left(V_{\lambda / \mu}\right)=W_{\lambda / \mu}$. It remains only to show that the set $\left\{e(\delta): \delta \in A_{\lambda / \mu}\right\}$ is linearly independent. Suppose that there is a linear relation

$$
\sum_{\delta \in A_{\lambda / \mu}} \alpha_{\delta} e(\delta)
$$

We want to show that $\alpha_{\delta}=0$ for all $\delta \in A_{\lambda / \mu}$. If not, then let $\delta_{0}$ be the standard matching that maximizes $T\left(\delta_{0}\right)$ among those $\delta \in A_{\lambda / \mu}$ with $\alpha_{\delta} \neq 0$.

In the proof of Lemma 2.12 below, we show that for any standard matching $\delta, e(\delta)$ has a non-zero $\delta$ coefficient in $V_{\lambda / \mu}$, and that if $\delta_{1}$ and $\delta_{2}$ are two standard matchings with $T\left(\delta_{1}\right) \prec T\left(\delta_{2}\right)$, then the $\delta_{2}$ coefficient of $e\left(\delta_{1}\right)$ is zero.

In particular, for any $\delta \in A_{\lambda / \mu}$ such that $T(\delta) \prec T\left(\delta_{0}\right)$, the $\delta_{0}$ coefficient of $e(\delta)$ is zero. Since the $\delta_{0}$ coefficient of $e\left(\delta_{0}\right)$ is not zero, we must have $\alpha_{\delta_{0}}=0$, contradicting our choice of $\delta_{0}$. Hence, we must have $\alpha_{\delta}=0$ for all $\delta \in A_{\lambda / \mu}$.

Another way to state Theorem 2.11 is that the columns of $P$ form a basis for the space $e\left(V_{\lambda / \mu}\right)$.

\subsection{Computation of $\operatorname{det} M$ and $\operatorname{det} \mathcal{M}$}

The computations of $\operatorname{det} M$ and $\operatorname{det} \mathcal{M}$ are virtually identical, so we will only compute $\operatorname{det} M$ explicitly, and merely state the corresponding result for $\operatorname{det} \mathcal{M}$. 
Suppose that $v \in V_{2 \nu}$ is an eigenvector of $T_{k}(x)$ with eigenvalue $h_{2 \nu}(x)$. Since $T_{k}(x)$ commutes with the action of $S_{\lambda / \mu}$, we have

$$
\begin{aligned}
T_{k}(x)(e(v)) & =e\left(T_{k}(x) v\right) \\
& =e\left(h_{2 \nu}(x) v\right) \\
& =h_{2 \nu}(x) e(v) .
\end{aligned}
$$

i.e. $e(v)$ is also an eigenvector of $T_{k}(x)$ with eigenvalue $h_{2 \nu}(x)$. Furthermore, it is not hard to see that the $h_{2 \nu}(x)$ are all distinct, so we must have $e(v) \in V_{2 \nu}$. We have shown that

$$
e\left(V_{2 \nu}\right) \subseteq V_{2 \nu}
$$

It now follows that

$$
\begin{aligned}
e\left(V_{\lambda / \mu}\right) & =e\left[\bigoplus_{2 \nu \vdash 2 k} V_{2 \nu}\right] \\
& =\bigoplus_{2 \nu \vdash 2 k} e\left(V_{2 \nu}\right) .
\end{aligned}
$$

Let $d_{\nu}=d_{\mu \nu}^{\lambda}$ be the dimension of $e\left(V_{\nu}\right)$ (thus, if $\nu$ is not even then $d_{\nu}=0$ ). For every even partition $\nu \vdash 2 k$, let $B^{\nu}=\left\{e\left(v_{1}^{\nu}\right), \ldots, e\left(v_{d_{\nu}}^{\nu}\right\}\right.$ be a basis for $e\left(V_{\nu}\right)$. Let $B_{\lambda / \mu}=\bigcup B^{\nu}$. We now have two bases for $e\left(V_{\lambda / \mu}\right)$, namely $e\left(A_{\lambda / \mu}\right)$, and $B_{\lambda / \mu}$.

Let $Q$ be the $F_{\lambda / \mu} \times B_{\lambda / \mu}$ matrix whose column indexed by $e\left(v_{i}^{\nu}\right)$ is the expansion of $e\left(v_{i}^{\nu}\right)$ in $V_{\lambda / \mu}$ in terms of the basis $F_{\lambda / \mu}$. Let $S$ be the $A_{\lambda / \mu} \times B_{\lambda / \mu}$ matrix such that

$$
P S=Q
$$

The matrix $S$ is an invertible transition matrix from the basis $e\left(A_{\lambda / \mu}\right)$, to the basis $B_{\lambda / \mu}$ for $e\left(V_{\lambda / \mu}\right)$.

The importance of the matrix $Q$ is the fact that its columns are eigenvectors for $T_{k}(x)$. Thus,

$$
\left(T_{k}(x) Q\right)_{e\left(v_{i}^{\nu}\right)}=h_{\nu}(x) Q_{e\left(v_{i}^{\nu}\right)} .
$$

This is equivalent to saying that $T_{k}(x) Q=Q D$, where $D$ is a $B_{\lambda / \mu} \times B_{\lambda / \mu}$ diagonal matrix whose diagonal entry in the column indexed by $e\left(v_{i}^{\nu}\right)$, is $h_{\nu}(x)$.

We can use these facts to study the product formulation of the matrix $M$ as follows:

$$
\begin{aligned}
S^{t} M & =S^{t} P^{t} T_{k}(x) J \\
& =Q^{t} T_{k}(x) J \\
& =D Q^{t} J \\
& =D S^{t} P^{t} J .
\end{aligned}
$$


From this we obtain

$$
\begin{aligned}
\operatorname{det} M & =\operatorname{det} D \operatorname{det}\left(P^{t} J\right) \\
& =\operatorname{det}\left(P^{t} J\right) \prod_{2 \nu \vdash 2 k} h_{2 \nu}(x)^{d_{2 \nu}} .
\end{aligned}
$$

Let $N=P^{t} J$. Then, $N$ is a $A_{\lambda / \mu} \times A_{\lambda / \mu}$ matrix, and $\operatorname{det}(N)$ is a scalar because the matrices $P$ and $J$ both have entries in $\mathbb{R}$. It is not hard to see that the $\left(\delta_{i}, \delta_{j}\right)$ entry of $N$ is the coefficient of $\delta_{j}$ in $e\left(\delta_{i}\right)$. We have

$$
\begin{aligned}
N_{i j}=N_{\delta_{i}, \delta_{j}} & =\sum_{\sigma \in C_{\lambda / \mu}} \sum_{\tau \in R_{\lambda / \mu}} \operatorname{sgn}(\sigma) \chi\left(\sigma \tau \delta_{i}=\delta_{j}\right) \\
& =\sum_{\sigma \in C_{\lambda / \mu}} \sum_{\tau \in R_{\lambda / \mu}} \operatorname{sgn}(\sigma) \chi\left(\tau \delta_{i}=\sigma \delta_{j}\right)
\end{aligned}
$$

where $\chi($ statement $)=1$ if the statement is true and 0 otherwise.

Notation. Let $R_{\delta}$ (resp. $C_{\delta}$ ) be the subgroup of $R_{\lambda / \mu}$ (resp. $C_{\lambda / \mu}$ ) that fixes the standard matching $\delta$.

Lemma 2.12.

$$
\operatorname{det} N=\prod_{\delta \in A_{\lambda / \mu}}\left|R_{\delta}\right|\left|C_{\delta}\right| .
$$

Proof. Renumber the standard matchings so that they appear in increasing order with respect to $\prec$. Note that for any row permutation $\tau$ of $[\lambda / \mu]$, and any matching $\epsilon$ of shape $\lambda / \mu, \overline{T(\tau \epsilon)}=\overline{T(\epsilon)}$.

If $N_{i j} \neq 0$, then for some $\tau \in R_{\lambda / \mu}$, and some $\sigma \in C_{\lambda / \mu}$, we have $\tau \delta_{i}=\sigma \delta_{j}$. By Theorem 2.5 we have

$$
\begin{aligned}
\overline{T\left(\delta_{j}\right)} & \preceq \overline{T\left(\sigma \delta_{j}\right)} \\
& =\overline{T\left(\tau \delta_{i}\right)} \\
& =\overline{T\left(\delta_{i}\right)} .
\end{aligned}
$$

But if $i<j$, then $\overline{T\left(\delta_{i}\right)} \prec \overline{T\left(\delta_{j}\right)}$, which contradicts $(2.50)$, so $N_{i j}=0$. In other words, $N$ is lower triangular.

Suppose that for some $\tau \in R_{\lambda / \mu}$, and some $\sigma \in C_{\lambda / \mu}$, we have

$$
\tau \delta_{i}=\sigma \delta_{i}
$$

If $\sigma \notin C_{\delta_{i}}$, then by Theorem 2.5,

$$
\overline{T\left(\sigma \delta_{i}\right)} \succ \overline{T\left(\delta_{i}\right)} .
$$

For all $\tau \in R_{\lambda / \mu}$

$$
\overline{T\left(\tau \delta_{i}\right)}=\overline{T\left(\delta_{i}\right)} .
$$


We conclude that if $\sigma \notin C_{\delta_{i}}$, then $\overline{T\left(\tau \delta_{i}\right)} \neq \overline{T\left(\sigma \delta_{i}\right)}$ for any $\tau \in R_{\lambda / \mu}$. Hence, if $\sigma \notin C_{\delta_{i}}$, then (2.51) cannot occur. If $\sigma \in C_{\delta_{i}}$, then (2.51) holds if and only if $\tau \in R_{\delta_{i}}$. Since all permutations in $C_{\delta_{i}}$ are even, we have

$$
N_{i i}=\left|R_{\delta_{i}}\right|\left|C_{\delta_{i}}\right|
$$

The Lemma clearly follows.

Lemma 2.13. $d_{\mu 2 \nu}^{\lambda}=d_{2 \nu}$ is equal to the Littlewood-Richardson coefficient $c_{\mu}^{\lambda} 2 \nu$.

Proof. We begin with the fact that the operator $e$ affords a skew representation of the symmetric group $S_{\lambda / \mu}$. More explicitly,

$$
e S_{\lambda / \mu} \cong S^{\lambda / \mu}=\bigoplus_{\eta \vdash 2 k} c_{\mu \eta}^{\lambda} S^{\eta}
$$

where $S^{\eta}$ is the irreducible Specht module indexed by $\eta$. It is a well known fact (see $[\mathrm{M}]$ ) that the coefficient $c_{\mu \eta}^{\lambda}$ is equal to the number of LR fillings of shape $\lambda / \mu$ whose Hebrew word has weight $\eta$ (see Definition 3.6).

The following are isomorphisms of vector spaces.

$$
\begin{aligned}
e\left(V_{2 \nu}\right) & \cong e S_{\lambda / \mu}\left(V_{2 \nu}\right) \\
& \cong e S_{\lambda / \mu} \otimes_{S_{\lambda / \mu}} V_{2 \nu} \\
& \cong \bigoplus_{\eta \vdash 2 k} c_{\mu \eta}^{\lambda} S^{\eta} \otimes_{S_{\lambda / \mu}} V_{2 \nu}
\end{aligned}
$$

But Schur's Lemma says that $S^{\eta} \otimes_{S_{\lambda / \mu}} V_{2 \nu}$ is one dimensional if $\eta=2 \nu$, and zero dimensional otherwise. Thus,

$$
e\left(V_{2 \nu}\right) \cong c_{\mu 2 \nu}^{\lambda} S^{\nu} \otimes_{S_{\lambda / \mu}} V_{2 \nu}
$$

That is, $e\left(V_{2 \nu}\right)$ has dimension $c_{\mu 2 \nu}^{\lambda}$, which is what we wanted to show.

Lemma 2.13, together with Lemma 2.12 and equation (2.48) completes the proof of the main theorem of this paper.

\section{Theorem 2.14.}

$$
\operatorname{det} M=\prod_{\delta \in A_{\lambda / \mu}}\left|R_{\delta}\right|\left|C_{\delta}\right| \prod_{2 \nu \vdash 2 k} h_{2 \nu}(x)^{c_{\mu 2 \nu}^{\lambda}} .
$$

Corollary 2.15 (Jockusch's Conjecture). det $M$ has only integer roots.

Proof. This is immediate from Theorem 2.14, since the polynomials $h_{2 \nu}(x)$ have only integer roots.

The arguments in this section can be used nearly word for word to prove the following generalization of Theorem 2.14. The only changes that need to be made are $M \rightarrow \mathcal{M}, T_{k}(x) \rightarrow \mathcal{T}_{k}\left(y_{1}, \ldots, y_{n}\right)$ and $h_{2 \nu}(x) \rightarrow z_{\nu}\left(y_{1}, \ldots, y_{n}\right)$. 
Theorem 2.16.

$$
\operatorname{det} \mathcal{M}=\prod_{\delta \in A_{\lambda / \mu}}\left|R_{\delta}\right|\left|C_{\delta}\right| \prod_{2 \nu \vdash 2 k} z_{\nu}\left(y_{1}, \ldots, y_{n}\right)^{c_{\mu}^{\lambda} 2 \nu} .
$$

Example 2.3. Although the determinants of these matrices are nice, in general their eigenvalues are not. The shape $(4,2) /(2)$, which is in some sense the smallest non-trivial example (because there are two standard matchings of this shape) already has eigenvalues that aren't nice. Here are the matrices one gets for that shape. The reader can verify that the determinants factor according to theorems 2.14 and 2.16, but that this is not reflected in the eigenvalues.

$$
\begin{gathered}
M=\left[\begin{array}{cc}
4 x^{2} & 4 x \\
4 x & 2 x^{2}+2 x
\end{array}\right] \\
\mathcal{M}=\left[\begin{array}{cc}
4 p_{1}^{2} & 4 p_{2} \\
4 p_{2} & 2 p_{1}^{2}+2 p_{2}
\end{array}\right]
\end{gathered}
$$

\section{Jeu de Taquin for standard matchings}

\subsection{Definition of the algorithm}

Assume that $D$ is a skew shape with one box $z$ removed (note that any skew shape can be considered a skew shape with one box removed by simply attaching a corner either on the Northwest or Southeast edges and then removing that box. Thus these definitions and results apply to plain skew shapes as well). Suppose $\delta$ is a standard matching of $D$. Denote the box to the right of $z$ by $x$, and the box below by $y$. Assume that either $x \in D$ or $y \in D$. We define a Northwest Jeu de Taquin (NW-JdT) move (for matchings) of $\delta$ at $z$ as follows.

If only one of $x, y$ lies in $D$ then let $w$ be that one. If both lie in $D$ then let $w$ be the element of $\{x, y\}$ that minimizes $\delta(w)$ with respect to $<_{h}$.

Now, define

$$
D^{\prime}=(D \backslash\{w\}) \cup\{z\},
$$

and define $\delta^{\prime}$, a matching for $D^{\prime}$, by

$$
\delta^{\prime}(b)= \begin{cases}\delta(b) & b \neq z, \delta(w) \\ \delta(w) & b=z \\ z & b=\delta(w) .\end{cases}
$$

In terms of 1-factors, $\delta^{\prime}$ has an edge between the boxes $z$ and $\delta(w)=\delta^{\prime}(z)$, whereas $\delta$ has an edge between $w$ and $\delta(w)$. This one edge "moves," and all the others remain fixed. The move $\delta \rightarrow \delta^{\prime}$ is called a NW-JdT move of $\delta$ at $z$.

Example 3.1. The following is a sequence of NW-JdT moves starting with a standard matching of shape $(6,4,2 / 2)$ and ending with a standard matching of shape $(6,3,2 / 1)$. The shaded box is the box $z$ removed from the skew shape. 


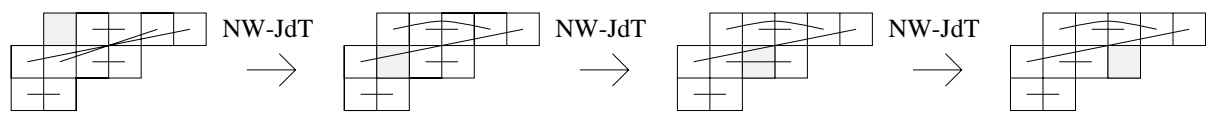

A SE-JdT move of $\delta$ is defined similarly. In this case, let $x$ be the box above $z$, and $y$ the box to the left of $z$. Assume that either $x \in D$ or $y \in D$. If only one of $x, y$ lies in $D$ then let $w$ be that one. If both lie in $D$ then let $w$ be the element of $\{x, y\}$ that maximizes $\delta(w)$ with respect to $<_{h}$. Use equations (3.1) and (3.2) to define $D^{\prime}$ and $\delta^{\prime}$. The move $\delta \rightarrow \delta^{\prime}$ is called a SE-JdT move of $\delta$ at $z$.

For a diagram $D$ with $n$ boxes, number the boxes $1 \ldots n$ in increasing order with respect to $<_{h}$. We will identify each box in $D$ with the number assigned to it (which is its position in the Hebrew order among the boxes of $D$ ). Under this identification the order $<_{h}$ is the same as the natural order on the integers $1,2, \ldots, n$, so $x<_{h} y$ and $x<y$ are equivalent. We will normally use $x<_{h}$ $y$, although there will be a few cases where the other notation will be more convenient.

Definition 3.1. A tableau $T$ of shape $D$ is a map $T: D \rightarrow \mathbb{Z}$. The Hebrew word for $T, h_{T}$, is the word $T(1) T(2) \ldots T(n)$, and the row word for $T, \pi_{T}$, is the reverse of $h_{T}$.

Definition 3.2. A tableau $T$ is called standard if $T$ is a bijection from $D$ to $\{1 \ldots n\}$, and $T$ increases along both rows and columns of $D$. If $D$ is the shape of a partition $\lambda \vdash n$, then $D$ is called a normal shape, and $T$ is called a standard Young tableau of normal shape.

Notice that for any tableau that is a bijection from $D$ to $\{1 \ldots n\}$, the Hebrew and row words are permutations of $\{1 \ldots n\}$ (in one-line form). In particular this is true for standard tableau and for matchings which we discuss below.

A matching $\epsilon$ for the diagram $D$ is a fixed point free involution $\epsilon: D \rightarrow D$. Under the identification of the boxes of $D$ with their Hebrew positions, this is equivalent to saying that $h_{\epsilon}$ is a fixed point free involution of $S_{n}$. Thus, we can think of matchings as tableaux whose Hebrew words are fixed point free involutions of $S_{n}$.

Lemma 3.1. Suppose $\epsilon$ is a matching of shape D, a skew shape with one box removed. Then $\epsilon$ is a standard matching if and only if $\epsilon$ is a standard tableau.

Proof. It is clear that if $\epsilon$ is a standard matching, then $\epsilon$ increases in both rows and columns.

Suppose now that $\epsilon$ is a standard tableau, and suppose $x<_{s} y \in D$. If $x$ and $y$ are in the same row or column, then the clearly $\epsilon(x)<\epsilon(y)$. If $x$ and $y$ are not in the same row or column, then let $z \in D$ be a corner of the rectangle $[x, y]_{<_{s}} \cap D$ such that $z \neq x, y$. There must be such a box $z$ since by Lemma 3.2 , the entire rectangle $[x, y]_{<_{s}}$ lies in the skew shape, and only one of the two corners that are not $x$ or $y$ could possibly be removed. Now, $z$ is in either the same row or column with both $x$ and $y$. So, we have $\epsilon(x)<\epsilon(z)<\epsilon(y)$. 
We have defined a JdT algorithm for standard matchings. There is also a well known JdT algorithm for standard tableaux (this is the algorithm originally named Jeu de Taquin; the algorithm defined here for standard matchings can be considered a modified version of the original). We describe this JdT algorithm now.

Assume that $D$ is a skew shape with one box $z$ removed, and $T$ is a standard tableau of shape of $D$. Denote the box to the right of $z$ by $x$, and the box below by $y$. Assume that either $x \in D$ or $y \in D$. We define a Northwest Jeu de Taquin (NW-JdT) move (for tableaux) of $T$ at $z$ as follows.

If only one of $x, y$ lies in $D$ then let $w$ be that one. If both lie in $D$ then let $w$ be the element of $\{x, y\}$ that minimizes $T(w)$.

Now, define

$$
D^{\prime}=(D \backslash\{w\}) \cup\{z\},
$$

and define $T^{\prime}$, a standard tableau for $D^{\prime}$ (this is easy to show), by

$$
T^{\prime}(b)= \begin{cases}T(b) & b \neq z \\ T(w) & b=z .\end{cases}
$$

A SE-JdT move of $T$ is defined similarly. In this case, let $x$ be the box above $z$, and $y$ the box to the left of $z$. Assume that either $x \in D$ or $y \in D$. If only one of $x, y$ lies in $D$ then let $w$ be that one. If both lie in $D$ then let $w$ be the element of $\{x, y\}$ that maximizes $T(w)$. Use equations (3.3) and (3.4) to define $D^{\prime}$ and $T^{\prime}$. The move $T \rightarrow T^{\prime}$ is called a SE-JdT move of $T$ at $z$.

Remark 3.1. Since we can regard any standard matching $\delta$ as a tableau, we can apply both JdT algorithms to $\delta$. We will be interested in comparing the outputs of these two algorithms. Note that if we perform NW-JdT moves on $\delta$, then both algorithms choose the same box $w$ to move. Similarly, both algorithms choose the same box to move in SW-JdT moves. This means that the output of these algorithms will be a matching and a tableau of the same shape $D^{\prime}$.

Example 3.2. We start with a standard matching of shape $(4,4,3,1 / 1)$ with the box $(2,2)$ removed. We then apply the two JdT algorithms to compare their outputs.

Note that the only difference between the outputs is that the numbers 6,7 and 8 are in different places. Note also that by doing the vertical move, we are changing the Hebrew positions of the boxes in the 6th, 7th, and 8th positions. As we will see later, this is not a coincidence.

\subsection{Jeu de Taquin preserves standardness}

The following Lemma is easily proved, and will be very useful in the proof of Theorem 3.3.

Lemma 3.2. For any skew shape $\lambda / \mu$, if $x<_{s} y$ are boxes in $[\lambda / \mu]$, then the entire interval $[x, y]_{<_{s}}$ is contained in $[\lambda / \mu]$. 


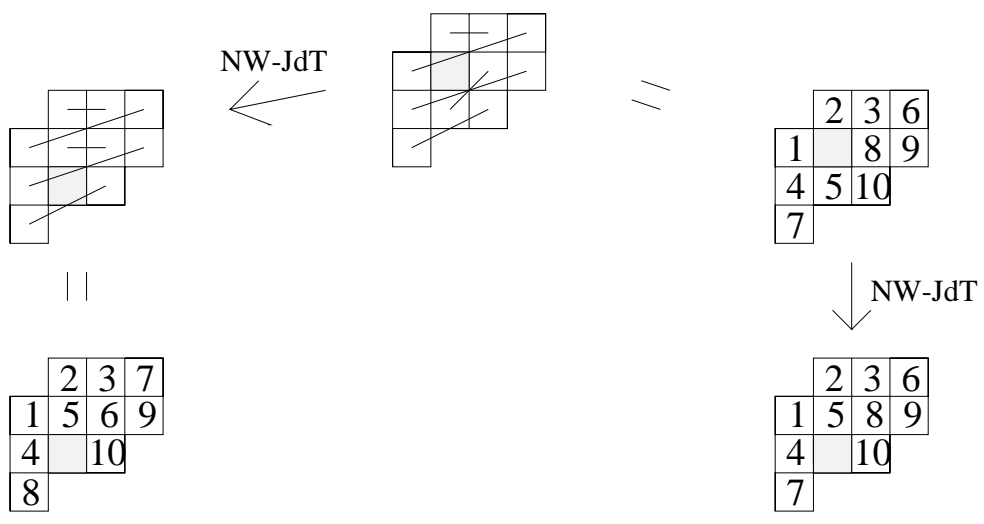

Theorem 3.3. Suppose $\delta$ is a standard matching of the diagram D, a skew shape with one box $z$ removed. If the matching $\delta^{\prime}$ of $D^{\prime}$ is obtained from $\delta$ by a $N W$-JdT or SE-JdT move for matchings at $z$, then $\delta^{\prime}$ is a standard matching for $D^{\prime}$.

Proof. We will prove this for a NW-JdT move. The proof for a SE-JdT move is essentially the same.

If $a \in D \cap D^{\prime}$, then it could happen that the Hebrew position of $a$ in $D$ is different from the Hebrew position of $a$ in $D^{\prime}$. The JdT algorithm for tableaux is "blind" to this possible difference, because a tableau for $D$ is simply a bijection from $D$ to $\{1, \ldots, n\}$, so the Hebrew order of the boxes is irrelevant. A matching however, is a bijection from $D$ to $D$. So, in the JdT algorithm for matchings, this possible difference has an effect which we need to understand.

Let $T^{\prime}$ be the tableau obtained from $\delta$ by a NW-JdT move for tableaux at $z$. Assume that $x$ is the box moved into position $z$ in both algorithms, and that $x$ is the box adjacent to $z$ and to the East. If this is the case, then clearly $h_{T^{\prime}}=h_{\delta}$, and so $h_{T^{\prime}}$ is a fixed point free involution. Thus, $T^{\prime}$ is a matching, and $T^{\prime}$ is obviously standard as well. Furthermore, the Hebrew positions of boxes in $D^{\prime}$ remain the same as they were in $D$, with $z$ taking on the Hebrew position in $D^{\prime}$ that $x$ occupied in $D$. This implies that if $b \neq z, \delta(x)$, then $\delta^{\prime}(b)=\delta(b)=T^{\prime}(b)$. Moreover, $\delta^{\prime}(\delta(x))=z$ and $\delta(\delta(x))=x$, and $z$ occupies in $D^{\prime}$ the same Hebrew position that $x$ occupies in $D$. Thus, numerically $\delta^{\prime}(\delta(x))=\delta(\delta(x))=T^{\prime}(\delta(x))$. Finally, $\delta^{\prime}(z)=\delta(x)=T^{\prime}(z)$. In other words, $\delta^{\prime}=T^{\prime}$. Therefore, $\delta^{\prime}$ is a standard matching, because $T^{\prime}$ is standard.

On the other hand, if $y$ is moved into position $z$, and $y$ is the box immediately below $z$, then the Hebrew position of each box in $(z, y)_{<_{n}}$ increases by one, and the Hebrew position of $z$ is the Hebrew position of $y$ minus the number of boxes 
THE ELECTRONIC JOURNAL OF COMBINATORICS 2 (1995),\#R23

in $(z, y)_{<_{h}}$. This means that $\delta^{\prime}$ is numerically given by

$$
\delta^{\prime}(b)= \begin{cases}\delta(b) & b \neq z, \delta(b) \notin(z, y]_{<_{h}}, \\ \delta(b)+1 & \delta(b) \in(z, y)_{<_{h}}, \\ \delta(y) & b=z, \\ y-\left|(z, y)_{<_{h}}\right| & \delta(b)=y .\end{cases}
$$

Compare this to $T^{\prime}$, for which

$$
T^{\prime}(b)= \begin{cases}\delta(b) & b \neq z, \delta(b) \notin(z, y]_{<_{h}}, \\ \delta(b) & \delta(b) \in(z, y)_{<_{h}} \\ \delta(y) & b=z \\ y & \delta(b)=y\end{cases}
$$

We can now compare $T^{\prime}$ to $\delta^{\prime}$ as follows:

$$
\delta^{\prime}(b)= \begin{cases}T^{\prime}(b) & \delta(b) \notin(z, y]_{<_{h}}, \\ T^{\prime}(b)+1 & \delta(b) \in(z, y)_{<_{h}}, \\ T^{\prime}(b)-\left|(z, y)_{<_{h}}\right| & \delta(b)=y .\end{cases}
$$

So, to get from $T^{\prime}$ to $\delta^{\prime}$, one "cycles" the set of numbers $\left\{\delta(b): \delta(b) \in(z, y]_{<_{h}}\right\}$. Since $T^{\prime}$ is standard, it is not hard to see that if $\delta^{\prime}$ is not standard, then the only place where standardness could be violated is at the box $\delta(y)$. Let $y^{\prime}=\delta(y)$. Since $\delta^{\prime}\left(y^{\prime}\right) \leq T^{\prime}\left(y^{\prime}\right)$, we conclude that if $y^{\prime}<_{s} b$, then

$$
\delta^{\prime}\left(y^{\prime}\right) \leq T^{\prime}\left(y^{\prime}\right)<T^{\prime}(b) \leq \delta^{\prime}(b) .
$$

It remains only to check that if $b<_{s} y^{\prime}$ then $\delta^{\prime}(b)<_{h} \delta^{\prime}\left(y^{\prime}\right)$. Let $c$ be the box immediately North of $y^{\prime}$, and $d$ the box immediately West of $y^{\prime}$. Clearly, it suffices to assume that $b \in\{c, d\}$.

If $y=c$ then $\delta$ has a vertical edge which cannot happen in a standard matching.

If $y=d$, then $c=x$ where $x$ is the box immediately to the East of $z$.

\begin{tabular}{|c|c|}
\hline $\mathrm{z}$ & $\mathrm{x}=\mathrm{c}$ \\
\hline $\mathrm{y}=\mathrm{d}$ & $\mathrm{y}^{\prime}$ \\
\hline
\end{tabular}

We have $\delta(x)<_{h} \delta\left(y^{\prime}\right)=y$, hence $\delta(x) \leq_{h} y^{\prime}=\delta(y)$ because $y^{\prime}$ is immediately East of $y$. But this contradicts the fact that we are making a vertical move $y \rightarrow z$, which implies that $\delta(y)<_{h} \delta(x)$. We conclude that $y \notin\{c, d\}$.

Since $\{c, d\} \cap\left\{y, y^{\prime}\right\}=\emptyset$, we have $\delta(c)=\delta^{\prime}(c)$ and $\delta(d)=\delta^{\prime}(d)$. We will assume that either

$$
\delta(c)=\delta^{\prime}(c)>_{h} \delta^{\prime}\left(y^{\prime}\right)=z,
$$


or

$$
\delta(d)=\delta^{\prime}(d)>_{h} \delta^{\prime}\left(y^{\prime}\right)=z,
$$

and find that these assumptions lead to a contradiction.

We need to define two sets contained in $D$. Let $A$ be the set of boxes in the same row as $z$ that are strictly West of $z$ and contained in $D$. Let $B$ be the set of boxes in the same row as $y$ that are strictly East of $y$ and contained in $D$. Observe that $A \cup B=(z, y)_{<_{h}} \cap D$. 


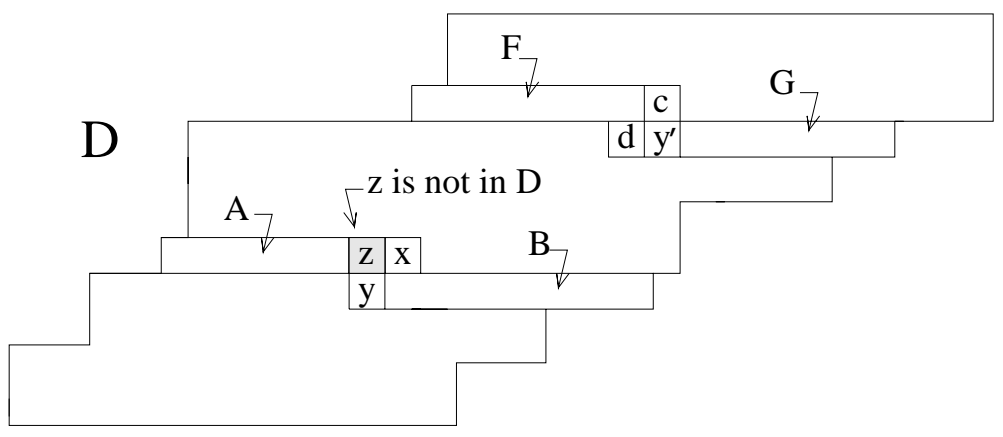

By the standardness of $\delta$, we know that $\delta(c), \delta(d)<_{h} \delta\left(y^{\prime}\right)=y$. This fact, together with (3.9) or (3.10) implies that either

$$
z<_{h} \delta(c)<_{h} y
$$

or

$$
z<_{h} \delta(d)<_{h} y \text {. }
$$

In other words, we must have $\delta(c) \in A \cup B$ or $\delta(d) \in A \cup B$. We now break into cases to show that these cannot happen.

Case 1: $\delta(c)$ or $\delta(d) \in B$ :

Suppose $f=\delta(d) \in B$. By Lemma 3.2, since $z$ and $f$ are in the skew shape, and $z<_{s} f, x$ is in the skew shape as well. Hence $x \in D$. But now, $x<_{s} f$ so we must have

$$
\delta(x)<_{h} \delta(f)=d .
$$

But this implies that $\delta(x) \leq_{h} y^{\prime}=\delta(y)$ a contradiction, since we are moving $y \rightarrow z$, and hence $\delta(y)<_{h} \delta(x)$. The same argument shows that $\delta(c) \notin B$.

Case 2: $\delta(d) \in A$ :

In this case $\delta(d)<_{s} y$, but $\delta(\delta(d))=d>_{h} y^{\prime}=\delta(y)$, contradicting the standardness of $\delta$.

Case 3: $\delta(c) \in A$ :

This case is more complicated. We will show that $\delta(c) \in A$ implies that there is an infinite sequence of distinct elements in $A$. Essentially, a cycle of elements in $D$ is created (some of which are in $A$ ) that can never end.

Before we begin with this part of the proof we need a little more notation. Define $F$ to be the set of boxes in the same row as $c$ that are strictly West of $c$ and contained in $D$. Let $G$ be the set of boxes in the same row as $y^{\prime}$ that are strictly East of $y^{\prime}$ and contained in $D$. We have $F \cup G=\left(c, y^{\prime}\right)_{<_{h}} \cap D$. 


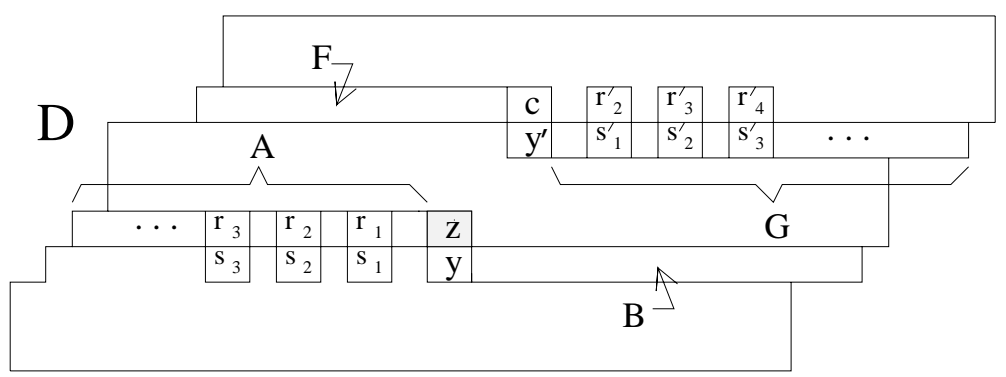

Let $r_{1}=\delta(c) \in A$, and let $s_{1}$ be the box directly below $r_{1}$. By Lemma 3.2, we have $s_{1} \in D$ (using $r_{1}<_{s} y$ ). Let $s_{1}^{\prime}=\delta\left(s_{1}\right)$. Since $r_{1}<_{s} s_{1}<_{s} y$ we must have

$$
c<_{h} s_{1}^{\prime}<_{h} y^{\prime},
$$

i.e. $s_{1}^{\prime} \in F \cup G$. But, if $s_{1}^{\prime} \in F$ then we have $s_{1}^{\prime}<_{s} c$, and $s_{1}>_{h} r_{1}=\delta(c)$ which contradicts the standardness of $\delta$. Thus, $s_{1}^{\prime} \in G$.

Now, for $i=2,3, \ldots$ we define the following: let $r_{i}^{\prime}$ be the box directly above $s_{i-1}^{\prime}$, let $r_{i}=\delta\left(r_{i}^{\prime}\right)$, let $s_{i}$ be the box directly below $r_{i}$ and let $s_{i}^{\prime}=\delta\left(s_{i}\right)$.

Claim. For all $i, r_{i} \in A$, and $s_{i}^{\prime} \in G$. Moreover,

$$
r_{1}<_{h} r_{2}<_{h} r_{3}<_{h} \cdots
$$

and

$$
s_{1}^{\prime}>_{h} s_{2}^{\prime}>_{h} s_{3}^{\prime}>_{h} \cdots
$$

Proof. We proceed by induction on $i$. We have already shown that $r_{1} \in A$ and $s_{1}^{\prime} \in G$. Suppose now that $r_{k} \in A$ and $s_{k}^{\prime} \in G$ for all $k<i$, and that $r_{1}<_{h} \cdots<_{h} r_{i-1}$, and $s_{1}^{\prime}>_{h} \cdots>_{h} s_{i-1}^{\prime}$.

First, note that since $s_{i-1}^{\prime} \in G \subseteq D$, by Lemma 3.2 , (using $c<_{s} s_{i-1}^{\prime}$ ) we have that $r_{i}^{\prime} \in D$. Note also that since $s_{i-2}^{\prime}>_{h} s_{i-1}^{\prime}, r_{i}^{\prime}$ lies to the East of $r_{i-1}^{\prime}$. Thus, $r_{i-1}^{\prime}<_{s} r_{i}^{\prime}$. Now, since

$$
r_{i-1}^{\prime}<_{s} r_{i}^{\prime}<_{s} s_{i-1}^{\prime}
$$

we must have

$$
r_{i-1}<_{h} r_{i}<_{h} s_{i-1} .
$$

We cannot have $s_{i-1}<_{s} r_{i}$ because this would imply that $s_{i-1}^{\prime}<_{h} r_{i}^{\prime}$. Since $r_{i}^{\prime}$ lies North of $s_{i-1}^{\prime}$ this clearly can't happen. We conclude that $r_{i}$ must be in the same row as $r_{i-1}$ and strictly to the West of $r_{i-1}$, i.e. $r_{i} \in A$, and $r_{1}<_{h} \cdots<_{h} r_{i}$. 
THE ELECTRONIC JOURNAL OF COMBINATORICS 2 (1995),\#R23

Now, using Lemma 3.2 (with $r_{i}<_{s} y$ ) we conclude that $s_{i} \in D$. Also, we have $s_{i}<_{s} s_{i-1}$ because they are in the same row, and $s_{i}$ appears to the West of $s_{i-1}$. So, since

$$
r_{i}<_{s} s_{i}<_{s} s_{i-1}
$$

we must have

$$
r_{i}^{\prime}<_{h} s_{i}^{\prime}<_{h} s_{i-1}^{\prime} .
$$

We cannot have $s_{i}^{\prime}<_{s} r_{i}^{\prime}$ because this would imply that $s_{i}<_{h} r_{i}$. Since $r_{i}$ lies North of $s_{i}$ this clearly can't happen. We conclude that $s_{i}^{\prime}$ must be in the same row as $s_{i-1}^{\prime}$ and strictly to the East of $s_{i-1}^{\prime}$, i.e. $s_{i}^{\prime} \in G$, and $s_{1}^{\prime}>_{h} \cdots>_{h} s_{i}^{\prime}$.

The claim just proved is an obvious contradiction because it implies that the sizes of $A$ and $G$ are infinite. So, $\delta^{\prime}(c), \delta^{\prime}(d)<_{h} \delta^{\prime}\left(y^{\prime}\right)$ as desired.

We have shown that $a<_{s} b \in D^{\prime}$ implies that $\delta^{\prime}(a)<_{h} \delta^{\prime}(b)$, which means that $\delta^{\prime}$ is a standard matching for $D^{\prime}$.

\subsection{Dual Knuth equivalence with JdT for tableaux}

Our objective in this section is to describe how the outputs of the two JdT algorithms are related. It turns out that this relationship can be expressed in terms of the dual Knuth equivalence for $S_{n}$.

The dual Knuth equivalence for $S_{n}$ is defined as follows. Let $\sigma, \tau \in S_{n}$. They differ by a dual Knuth relation of the first kind if for some $k$

$$
\sigma=\ldots k+1 \ldots k \ldots k+2 \ldots
$$

and

$$
\tau=\ldots k+2 \ldots k \ldots k+1 \ldots
$$

They differ by a dual Knuth relation of the second kind if for some $k$

$$
\sigma=\ldots k \ldots k+2 \ldots k+1 \ldots
$$

and

$$
\tau=\ldots k+1 \ldots k+2 \ldots k \ldots
$$

The permuations $\sigma$ and $\tau$ are dual Knuth equivalent, (written $\sigma \cong{ }^{*} \tau$ ) if one can get from $\sigma$ to $\tau$ via a sequence of dual Knuth relations of the first and second kinds.

The following easy Lemma will simplify the proof of the Theorem 3.5.

Lemma 3.4. Suppose $a, s \in \mathbb{Z}^{+}$. Then

$$
a+1 \quad a \quad a+2 \cdots a+s \cong K^{*} a+s \quad a \quad a+1 \cdots a+s-1,
$$

and

$$
a+1 \cdots a+s-1 \quad a+s+1 \quad a+s \cong{ }^{*} a+2 \cdots a+s \quad a+s+1 \quad a+1 .
$$


Theorem 3.5. Suppose $\delta$ is a standard matching for $D$, where $D$ is a skew shape with one box $z$ removed. Let $T^{\prime}$ be the standard tableau for $D^{\prime}$ obtained by performing a $N W$-JdT (resp. SE-JdT) move for tableaux on $\delta$ at $z$. Let $\delta^{\prime}$ be the standard matching for $D^{\prime}$ obtained by performing a NW-JdT (SE-JdT) move for matchings on $\delta$ at $z$. Then $\pi_{T^{\prime}} \cong K^{*} \pi_{\delta^{\prime}}$.

Proof. We prove this for a NW-JdT move. The proof for a SE-JdT move is essentially the same.

Assume that $x$ is the box moved into position $z$ in both JdT algorithms, and that $x$ is the box adjacent to $z$ and to the East. If this is the case, then the Hebrew positions of the boxes in $D^{\prime}$ remain the same as they were in $D$, with $z$ taking on the Hebrew positon in $D^{\prime}$ that $x$ had in $D$. Clearly, in this case, we have $\pi_{\delta}=\pi_{\delta^{\prime}}=\pi_{T^{\prime}}$, so $\pi_{T^{\prime}} \cong K^{*} \pi_{\delta^{\prime}}$ trivially.

On the other hand, if $y$ is moved into position $z$, and $y$ is the box immediately below $z$, then the Hebrew position of each box in $(z, y)_{<_{h}}$ increases by one, and the Hebrew position of $z$ is the Hebrew position of $y$ minus the number of boxes in $(z, y)_{<_{h}}$. This means that $\delta^{\prime}$ is numerically given by

$$
\delta^{\prime}(b)= \begin{cases}\delta(b) & b \neq z, \delta(b) \notin(z, y]_{<_{h},} \\ \delta(b)+1 & \delta(b) \in(z, y)_{<_{h}}, \\ \delta(y) & b=z, \\ y-\left|(z, y)_{<_{h}}\right| & \delta(b)=y .\end{cases}
$$

Compare this to $T^{\prime}$, for which

$$
T^{\prime}(b)= \begin{cases}\delta(b) & b \neq z, \\ \delta(y) & b=z .\end{cases}
$$

Observe that the places where $T^{\prime}$ and $\delta^{\prime}$ can differ are exactly those $b \in D^{\prime}$ that satisfy $\delta(b) \in(z, y]_{<_{h}}$. Thus, the row words $\pi_{T^{\prime}}$ and $\pi_{\delta^{\prime}}$ are the same except at the positions corresponding to those $b \in D^{\prime}$ such that $\delta(b) \in(z, y]_{<_{h}}$.

We define four subsets of $D^{\prime}$ as follows:

Let $B$ be the set of boxes in $D$ strictly West of $z$ and in the same row as $z$. Let $G$ be the set of boxes in $D$ that have a box in $B$ immediately above.

Let $F$ be the set of boxes in $D$ strictly East of $x$ and in the same row as $x$. Let $A$ be the set of boxes in $D$ that have a box in $F$ immediately below.

Write

$$
\begin{aligned}
& A=\left\{a_{1}<_{h} a_{2}<_{h} \cdots<_{h} a_{r}\right\} \\
& B=\left\{b_{1}<_{h} b_{2}<_{h} \cdots<_{h} b_{l}\right\} \\
& F=\left\{f_{1}<_{h} f_{2}<_{h} \cdots<_{h} f_{r}\right\} \\
& G=\left\{g_{1}<_{h} g_{2}<_{h} \cdots<_{h} g_{l}\right\}
\end{aligned}
$$

Note that $B \cup F=(z, y)_{<_{h}}$, and observe that $M=A \cup B \cup F \cup G \cup\{z\}$ is a $<_{h}$ interval in $D^{\prime}$. By the comments above, if $\delta(b) \notin M$, then numerically, 
THE ELECTRONIC JOURNAL OF COMBINATORICS 2 (1995),\#R23

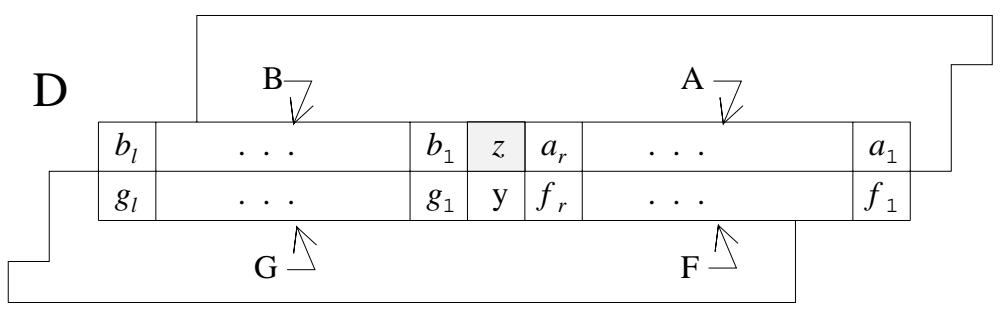

$\delta^{\prime}(b)=T^{\prime}(b)$. For convenience, we will shift the numbering of all the boxes in $D^{\prime}$ so that the boxes of $M$ are numbered $1 \ldots|M|$. So, the tableaux $T^{\prime}$ and $\delta^{\prime}$ may have nonpositive numbers in them, but the subsequences $\sigma_{T^{\prime}}$ of $\pi_{T^{\prime}}$, and $\sigma_{\delta^{\prime}}$ of $\pi_{\delta^{\prime}}$, corresponding to the positions $b$ with $\delta(b) \in M$, will be permutations of $\{1 \ldots|M|\}$.

We have the following inequalities:

$$
T^{\prime}\left(g_{1}\right)<T^{\prime}(z)<T^{\prime}\left(a_{r}\right)
$$

and

$$
\delta^{\prime}\left(g_{1}\right)<\delta^{\prime}(z)<\delta^{\prime}\left(a_{r}\right) .
$$

The only inequality that isn't obvious here is $\delta^{\prime}\left(g_{1}\right)<\delta^{\prime}(z)$. One can see this by noting that $\delta\left(g_{1}\right)<\delta(y)=\delta^{\prime}(z)$, and that $\delta^{\prime}\left(g_{1}\right) \leq \delta\left(g_{1}\right)+1$ (this follows from equations (3.23) and (3.24)), so we must have $\delta^{\prime}\left(g_{1}\right) \leq \delta^{\prime}(z)$. But equality is not allowed, so $\delta^{\prime}\left(g_{1}\right)<\delta^{\prime}(z)$.

Now, for any tableau $U$, if $c<_{h} d$, then $U(d)$ comes before $U(c)$ in the row word $\pi_{U}$. In our case, if $a \in B \cup G$ and $b \in A \cup F$, then we have

$$
\delta^{\prime}(a) \leq_{h} \delta^{\prime}\left(g_{1}\right)<_{h} \delta^{\prime}(z)<_{h} \delta^{\prime}\left(a_{r}\right) \leq_{h} \delta^{\prime}(b),
$$

which implies that $b=\delta^{\prime}\left(\delta^{\prime}(b)\right)$ occurs before $a=\delta^{\prime}\left(\delta^{\prime}(a)\right)$ in $\pi_{\delta^{\prime}}$, and $z$ occurs between them.

Furthermore, $\delta^{\prime}\left(b_{i}\right)<_{h} \delta^{\prime}\left(g_{i}\right)$ so $g_{i}$ occurs before $b_{i}$ in $\pi_{\delta^{\prime}}$. Similarly, $f_{i}$ occurs before $a_{i}$ in $\pi_{\delta^{\prime}}$. Finally, the $a_{i}$ 's, $b_{i}$ 's, $f_{i}$ 's and $g_{i}$ 's all occur in increasing order of their subscripts. So, the subsequence $\sigma_{\delta^{\prime}}$ looks like

$$
\sigma_{\delta^{\prime}}=\begin{aligned}
& f_{1} \ldots f_{r} \\
& a_{1} \ldots a_{r}
\end{aligned} ; \begin{aligned}
& g_{1} \ldots g_{l} \\
& b_{1} \ldots b_{l}
\end{aligned}
$$

where the notation used is interpreted as follows: if $a$ sits directly above $b$ then $a$ comes before $b$. If $a$ is to the left of $b$ and in the same row as $b$, then $a$ comes before $b$. All $f_{i}$ 's and $a_{i}$ 's come before $z$. All $g_{i}$ 's and $b_{i}$ 's come after $z$.

Example 3.3.

$$
7812934105116=\begin{array}{llllcc}
7 & 8 & 9 & & 10 & 11 \\
1 & 2 & 3 & 4 & 6
\end{array}
$$


THE ELECTRONIC JOURNAL OF COMBINATORICS 2 (1995),\#R23

whereas

$$
7128934101156 \neq \begin{array}{ccccc}
7 & 8 & 9 & 10 & 11 \\
1 & 2 & 3 & 5 & 6
\end{array}
$$

because 2 comes before 8 .

Of course, there are many permutations that are of this form, so in the future when we have an equivalence between two permutations denoted this way, we will always assume that the order in which we read off the letters for the two permutations is the same.

Numerically we have

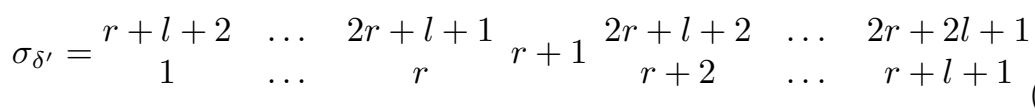

Using equations (3.23), (3.24) and (3.29), we find that

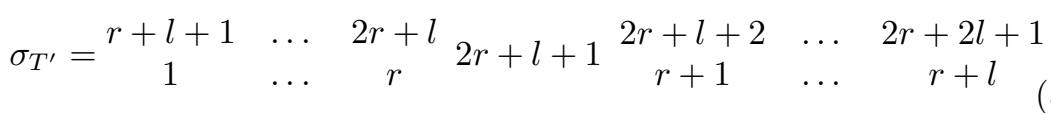

To prove the theorem, it suffices to show that $\sigma_{T^{\prime}} \cong K^{*} \sigma_{\delta^{\prime}}$. We do this by induction on $r+l$. If $r+l=0$, then $\sigma_{T^{\prime}}=1=\sigma_{\delta^{\prime}}$, and the theorem is trivially true.

If $l>0$ then both permutations have letters to the right of the "middle" position. Using Lemma 3.4 (the sequence where the Lemma is used is underlined), we obtain

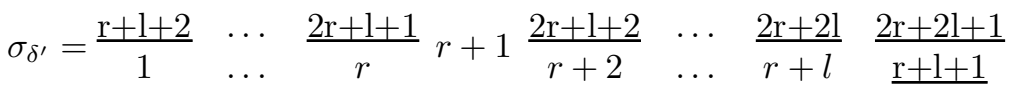

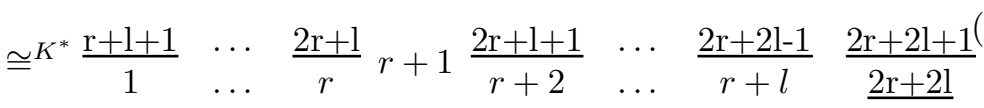

Now, by induction in the first step, and Lemma 3.4 in the second, we have

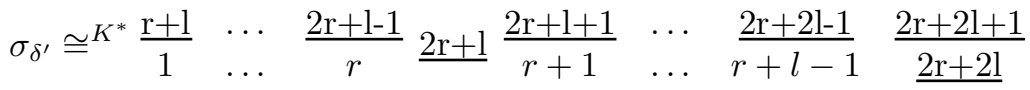

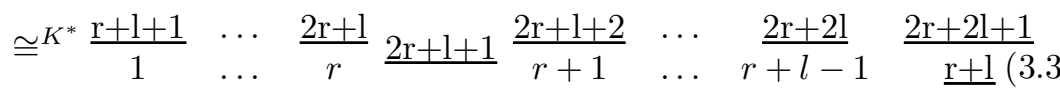

$$
\begin{aligned}
& =\sigma_{T^{\prime}} .
\end{aligned}
$$

A symmetric argument proves that $\sigma_{\delta^{\prime}} \cong K^{*} \sigma_{T^{\prime}}$ if $r>0$. 
THE ELECTRONIC JOURNAL OF COMBINATORICS 2 (1995),\#R23

\subsection{The normal shape obtained via JdT}

Before stating the main theorem of this section, some discussion of the RobinsonSchensted-Knuth insertion algorithm (RSK) is necessary. What we do here will leave out many details (including the definition of RSK) and will focus only on what is needed. We will consider only row insertion, so RSK means RSK row insertion (and not column insertion) in the discussion below.

RSK provides a bijection between elements of $S_{n}$ and pairs $(P, Q)$ of standard Young tableau of the same shape (having $n$ boxes) $[S]$. Denote the pair obtained from the permutation $\sigma$ by $(P(\sigma), Q(\sigma))$.

We will need several standard facts about RSK, JdT for tableaux, and dual Knuth equivalence which we state below. For a more complete discussion, see Sagan's book [Sa].

Fact 3.6. $R S K$ applied to $\sigma^{-1}$ produces the same pair of tableaux that are produced by $\sigma$ in the reverse order, i.e. $P\left(\sigma^{-1}\right)=Q(\sigma)$ and $Q\left(\sigma^{-1}\right)=P(\sigma)$. Also, if $\sigma^{r}$ is the reverse of $\sigma$ then $P\left(\sigma^{r}\right)=P(\sigma)^{t}$.

In particular, this implies that if $\sigma$ is an involution in $S_{n}$, then $P(\sigma)=Q(\sigma)$. In fact, RSK provides a bijection between the set of involutions of $S_{n}$ and the set of standard tableau of all normal shapes $\lambda \vdash n$. This is refined even further by the following fact which was proved by Schützenberger.

Fact 3.7. [Sch] Let $0 \leq k \leq n$ be fixed. Then $\sigma \mapsto P(\sigma)$ is a bijective map between involutions of $S_{n}$ with $k$ fixed points, and standard tableaux of all normal shapes $\lambda \vdash n$ such that $\lambda$ has exactly $k$ columns of odd length.

Fact 3.8. Let $T$ be a standard tableau of shape $D$, which is a skew shape with one box removed. If we use JdT to bring $T$ to some standard tableau of normal shape $T^{\prime}$, then $T^{\prime}=P\left(\pi_{T}\right)$. Hence, if one can get from the standard tableau $T$ to the standard tableau $T^{\prime \prime}$ via JdT, then $P\left(\pi_{T^{\prime \prime}}\right)=P\left(\pi_{T}\right)$.

Fact 3.9. If $\sigma$ and $\tau$ are elements of $S_{n}$, then $\sigma \cong K^{*} \tau$ if and only if $Q(\sigma)=$ $Q(\tau)$.

Theorem 3.10. Suppose $\delta$ is a standard matching of shape D, a skew shape with one box $z$ removed. If we use JdT for tableaux to bring $\delta$ to a standard tableau of normal shape $\nu_{1}$, and we apply JdT for matchings to bring $\delta$ to a standard matching of normal shape $\nu_{2}$, then $\nu_{1}=\nu_{2}$. Moreover, $\nu_{1}$ (and hence $\left.\nu_{2}\right)$ is even.

Proof. We show first that $\nu_{1}$ is even. By Fact $3.8, \nu_{1}=$ shape $P\left(\pi_{\delta}\right)$. Also, $\pi_{\delta}$ is the reverse of the Hebrew word $h_{\delta}$ which is a fixed point free involution in $S_{2 n}$. Thus, by Fact 3.7, the columns of $P\left(h_{\delta}\right)$ all have even length. Finally, Fact 3.6 implies that $\nu_{1}=$ shape $P\left(\pi_{\delta}\right)=$ shape $P\left(h_{\delta}\right)^{t}$, and therefore every row of $\nu_{1}$ has even length.

Let $T^{\prime}$ be the standard tableau for $D^{\prime}$ obtained by a JdT move for tableaux at $z$, and let $\delta^{\prime}$ be the standard matching for $D^{\prime}$ obtained by a JdT move 
for matchings at $z$. The following are consequences of Facts 3.8 and 3.9, and Theorem 3.5.

$$
\begin{aligned}
P\left(\pi_{\delta}\right) & =P\left(\pi_{T^{\prime}}\right) \\
Q\left(\pi_{T^{\prime}}\right) & =Q\left(\pi_{\delta^{\prime}}\right) .
\end{aligned}
$$

These imply the following string of equalities:

$$
\text { shape } \begin{aligned}
P\left(\pi_{\delta}\right) & =\text { shape } P\left(\pi_{T^{\prime}}\right) \\
& =\operatorname{shape} Q\left(\pi_{T^{\prime}}\right) \\
& =\operatorname{shape} Q\left(\pi_{\delta^{\prime}}\right) \\
& =\operatorname{shape} P\left(\pi_{\delta^{\prime}}\right) .
\end{aligned}
$$

From this, we conclude that if $\delta^{\prime}$ is obtained from $\delta$ using JdT for matchings, then shape $P\left(\pi_{\delta^{\prime}}\right)=$ shape $P\left(\pi_{\delta}\right)$. In particular, suppose that $\delta^{\prime}$ is a standard matching of normal shape $\nu_{2}$. In that case

$$
\begin{aligned}
\nu_{2} & =\text { shape } \delta^{\prime} \\
& =\operatorname{shape} P\left(\pi_{\delta^{\prime}}\right) \\
& =\operatorname{shape} P\left(\pi_{\delta}\right) \\
& =\nu_{1} .
\end{aligned}
$$

Here, the second equality comes from the fact that since shape $\delta^{\prime}$ is normal, Fact 3.8 implies that we must have $P\left(\pi_{\delta^{\prime}}\right)=\delta^{\prime}$.

Corollary 3.11. If the standard matching $\delta$ is taken to a standard matching $\delta^{\prime}$ for a normal even shape $\nu$ via $J d T$, then $\delta^{\prime}$ is independent of the sequence of $J d T$ moves chosen. In fact, $\nu=$ shape $P\left(\pi_{\delta}\right)$, and $\delta^{\prime}$ is the unique standard matching of shape $\nu$.

Proof. This follows from Theorem 3.10 and Corollary 2.4.

Because of Corollary 3.11, we can make the following definition.

Definition 3.3. If $\delta$ is a standard matching, then let $\nu(\delta)$ denote the shape of the standard matching of normal shape obtained from $\delta$ via JdT.

For the normal shape $\nu \vdash 2 n$, define $g_{\mu \nu}^{\lambda}$ to be the number of times $\nu$ appears as $\nu(\delta)$ for some $\delta \in A_{\lambda / \mu}$. We would like to compute these numbers $g_{\mu \nu}^{\lambda}$. For this, we need a modified version of a result of Dennis White. This version follows easily from the main result in $[\mathrm{Wh}]$. Before we state the theorem, we need to make some definitions.

Definition 3.4. A word $w=w_{1} w_{2} \ldots w_{n}$ in the alphabet $\mathbb{Z}^{+}$is called a lattice permutation if for every $j=1, \ldots, n$, the initial segment $w_{1} \ldots w_{j}$ of $w$ contains at least as many $i$ 's as it does $(i+1)^{\prime} s$, for every $i \in \mathbb{Z}^{+}$. The weight of $w$ is the vector $\left(v_{1}, \ldots, v_{n}\right)$, where $v_{i}$ is the number of $i$ 's in $w$. Clearly, the weight of a lattice permutation is always a partition. 
Definition 3.5. If $T$ is any standard tableau of the normal shape $\nu \vdash n$, define $\operatorname{lp}(T)$ to be the word $w=w_{1} \ldots w_{n}$, where $w_{i}$ is the row in which $i$ appears in $T$. Clearly, $\operatorname{lp}(T)$ is a lattice permutation of weight $\nu$. On the other hand, given a lattice permutation $w$ of weight $\nu$, there is a unique standard tableau $T$ of shape $\nu$, satisfying $\operatorname{lp}(T)=w$.

Definition 3.6. A tableau $T$ of shape $\lambda / \mu$ is called semistandard, if $T$ strictly increases down columns, and weakly increases from left to right along rows. A semistandard tableau $T$ is called a Littlewood-Richardson filling ( LR filling) of $T$, if $h_{T}$ is a lattice permutation. Let $c_{\mu \nu}^{\lambda}$ denote the number of LR fillings $T$ of shape $\lambda / \mu$ such that $h_{T}$ has weight $\nu$. It is a well known fact the the number $c_{\mu \nu}^{\lambda}$ is equal to the Littlewood-Richardson coefficient discussed in Lemma 2.13 $[\mathrm{M}]$.

Let $\omega_{0}$ denote the involution in $S_{n}$ with one line form $n n-1 \ldots 21$.

Theorem 3.12. [Wh] Let $\pi$ be any permutation in $S_{n}$, and $\lambda / \mu$ a skew shape of size $n$. Then $\operatorname{lp}(P(\pi))=h_{T}$ for some LR filling $T$ of shape $\lambda / \mu$ if and only if $\omega_{0} \pi^{-1}=h_{U}$ for some standard tableau $U$ of shape $\lambda / \mu$.

We need a few more standard facts about the RSK (row) insertion algorithm.

Fact 3.13. For any $\sigma \in S_{n}$, the following properties of RSK hold:

(1) $\left.\left(P\left(\omega_{0} \sigma \omega_{0}\right), Q\left(\omega_{0} \sigma \omega_{0}\right)\right)=\left(P(\sigma)_{\text {evac }}, Q(\sigma)_{\text {evac }}\right)\right)$, where $P_{\text {evac }}$ is obtained from $P$ by a process known as evacuation. This fact can be used as a definition of evacuation for standard tableaux.

(2) $\left(P_{\text {evac }}\right)^{t}=\left(P^{t}\right)_{\text {evac }}$.

(3) $[S c h]\left(P_{\text {evac }}\right)_{\text {evac }}=P$.

(4) shape $P_{\text {evac }}=$ shape $P$.

(5) $\left(P\left(\sigma \omega_{0}\right), Q\left(\sigma \omega_{0}\right)\right)=\left(P(\sigma)^{t}, Q(\sigma)_{\text {evac }}^{t}\right)$.

We can now state the Theorem which gives the values of the numbers $g_{\mu \nu}^{\lambda}$.

Theorem 3.14. For any skew shape $\lambda / \mu$ and any normal even shape $\nu$ with $|\lambda / \mu|=|\nu|$, we have $g_{\mu \nu}^{\lambda}=c_{\mu \nu}^{\lambda}$.

Proof. We find a bijection $\phi$, between the set of standard matchings $\delta$ with $\nu(\delta)=\nu$, and the set of LR fillings $T$ of shape $\lambda / \mu$ such that $h_{T}$ has weight $\nu$.

Suppose $\delta \in A_{\lambda / \mu}$, and $\nu(\delta)=\nu$. Then $h_{\delta}$ is a fixed point free involution of $S_{2 n}$ where $\lambda / \mu$ has size $2 n$. Furthermore, $h_{\delta}=\pi_{\delta} \omega_{0}$. Taking inverses, we obtain

$$
h_{\delta}=\omega_{0} \pi_{\delta}^{-1} .
$$

Since $\delta$ is standard tableau, by Theorem 3.12, we have $\operatorname{lp}\left(P\left(\pi_{\delta}\right)\right)=h_{T}$ for some LR filling $T$ of shape $\lambda / \mu$. Since shape $P\left(\pi_{\delta}\right)=\nu$, it follows that $h_{T}$ has weight $\nu$. We define $\phi(\delta)=T$. 
Now, if $T$ is a LR filling of shape $\lambda / \mu$ such that $h_{T}$ has weight $\nu$, then let $P$ be the standard tableau of shape $\nu$ such that $\operatorname{lp}(P)=h_{T}$. Let $\pi \in S_{2 n}$ be the permutation such that

$$
\begin{aligned}
& P(\pi)=P, \\
& Q(\pi)=P_{\text {evac }} .
\end{aligned}
$$

Using Fact 3.13 (5), (2) and (3), we obtain

$$
\begin{aligned}
& P\left(\pi \omega_{0}\right)=P^{t}, \text { and } \\
& Q\left(\pi \omega_{0}\right)=\left(P_{\text {evac }}\right)_{\text {evac }}^{t}=P^{t} .
\end{aligned}
$$

Since $P^{t}$ consists entirely of even columns, Facts 3.6 and 3.7 imply that $\pi \omega_{0}$ is a fixed point free involution in $S_{2 n}$. Thus,

$$
\pi \omega_{0}=\left(\pi \omega_{0}\right)^{-1}=\omega_{0} \pi^{-1} .
$$

We have $\operatorname{lp}(P(\pi))=h_{T}$, so by Theorem $3.12, \omega_{0} \pi^{-1}=h_{\delta}$ for some standard tableau $\delta$ of shape $\lambda / \mu$. Moreover, since $\omega_{0} \pi^{-1}$ is a fixed point free involution of $S_{2 n}, \delta$ is a matching of shape $\lambda / \mu$, and hence $\delta \in A_{\lambda / \mu}$. To show that $\nu(\delta)=\nu$, we compute

$$
\begin{aligned}
\nu(\delta) & =\operatorname{shape} P\left(\pi_{\delta}\right) \\
& =\operatorname{shape} P\left(h_{\delta} \omega_{0}\right) \\
& =\operatorname{shape} P\left(\omega_{0} \pi^{-1} \omega_{0}\right) \\
& =\operatorname{shape} P\left(\pi^{-1}\right)_{\mathrm{evac}} \\
& =\operatorname{shape} P\left(\pi^{-1}\right) \\
& =\operatorname{shape} Q(\pi) \\
& =\nu .
\end{aligned}
$$

Clearly, $\phi(\delta)=T$. In fact, it is not hard to see that $\phi$ is a bijection, which finishes the proof of the theorem.

\subsection{An alternate statement of the main theorem}

Theorem 3.14 allows us to restate Theorem 2.14 in terms of Jeu de Taquin for matchings as follows.

Theorem 3.15.

$$
\operatorname{det} M=\prod_{\delta \in A_{\lambda / \mu}}\left|R_{\delta}\right|\left|C_{\delta}\right| h_{\nu(\delta)}(x) .
$$

Similarly, we can restate Theorem 2.16.

\section{Theorem 3.16.}

$$
\operatorname{det} \mathcal{M}=\prod_{\delta \in A_{\lambda / \mu}}\left|R_{\delta}\right|\left|C_{\delta}\right| z_{1 / 2(\nu(\delta))}\left(y_{1}, \ldots, y_{n}\right)
$$


THE ELECTRONIC JOURNAL OF COMBINATORICS 2 (1995),\#R23

\section{References}

[Brr] R. Brauer, On algebras which are connected to the semisimple continuous groups, Ann. of Math. 38 (1937), 857-872.

[Brn] W. P. Brown, An algebra related to the orthogonal group, Michigan Math. J. 3 (1955-1956), 1-22.

[HW1] P. Hanlon and D. Wales, On the decomposition of Brauer's centralizer algebras, J. Algebra 121 (1989), 409-445.

[HW2] P. Hanlon and D. Wales, Eigenvalues connected with Brauer's centralizer Algebras, J. Algebra 121 (1989), 446-476.

[HW3] P. Hanlon and D. Wales, Computing the discriminants of Brauer's centralizer algebras, Math. Comput. 54, No. 190 (1990), 771-796.

[HW4] P. Hanlon and D. Wales, A Tower Construction for the Radical in Brauer's centralizer algebras, J. Algebra 164 (1994), 773-830.

[Ja1] A. T. James, The distribution of the latent roots of the covariance matrix, Ann. Math. Statist. 31 (1960), 151-158.

[Ja2] A. T. James, Zonal polynomials of the real positive definite symmetric matrices, Ann. of Math. 74 (1961), 456-469.

[Ja3] A.T. James, Distributions of matrix variates and latent roots derived from normal samples, Ann. Math. Statist. 35 (1964), 475-501.

[Jo] V. F. R. Jones, Index for Subfactors, Inv. Math. 72 (1983), 1-25.

$[\mathrm{M}] \quad$ I. G. Macdonald, "Symmetric Functions and Hall Polynomials," Clarendon, London, 1979.

[Sa] B. Sagan, "The Symmetric Group," Wadsworth \& Brooks/Cole Math. Series, Pacific Grove, CA, 1991.

[S] C. Schensted, Longest increasing and decreasing subsequences, Canad. J. Math. 13 (1961), 179-191.

[Sch] M.-P. Schützenberger, La correspondance de Robinson, in "Combinatoire et Représentation du Groupe Symétrique," Lecture Notes in Math., Vol. 579, Springer-Verlag, New York/Berlin, 1977.

[Wz] H. Wenzl, On the structure of Brauer's centralizer algebras, Ann. of Math. 128 (1988), 173-193.

[Wl] H. Weyl, "The Classical Groups," Princeton Univ. Press, Princeton, NJ, 1939.

[Wh] D. E. White, Some connections between the Littlewood-Richardson rule and the construction of Schensted, J. Combin. Theory Ser. A 30 (1981), 237-247. 